\section{Fueling Immunity: Insights into Metabolism and Lymphocyte Function}

Erika L. Pearce, ${ }^{*}$ Maya C. Poffenberger, Chih-Hao Chang, Russell G. Jones*

Background: Naïve lymphocytes circulate in the body in a resting state, but upon recognition of foreign antigen and receipt of proper costimulatory signals, these cells become activated, undergo a rapid burst in proliferation, and assume effector functions aimed at controlling or killing the invader. There is a growing appreciation that changes in peripheral T cell function are not only supported by but are dependent on metabolic reprogramming and that specific effector functions cannot proceed without adopting the correct metabolism. However, the reasons underlying why $T$ cells adopt specific metabolic programs and the impact that these programs have on $\mathrm{T}$ cell function and, ultimately, immunological outcome remain unclear.

Advances: Research into the metabolism of tumor cells has provided valuable insight into the metabolic pathways important for cell proliferation and survival, as well as the influence of metabolites themselves on signal transduction and epigenetic programming. Many of these concepts have shaped how we view metabolism in T cells. However, it is important to note that, unlike tumors, $T$ cells rapidly transition between resting catabolic states (naïve and memory $T$ cells) to one of growth and proliferation (effector T cells) as part of a normal developmental program. In addition, as T cells differentiate during an immune response, they also move from what are presumably nutrientreplete lymphoid organs to sites of cancer or infection, where oxygen, nutrients, and growth factors may become limiting. Thus, $T$ cells must metabolically adapt to these changing conditions in order to perform their necessary functions. In this Review, we highlight emerging areas in the metabolism of these dynamic cells and discuss the potential impact of metabolic control on T cell fate, plasticity, and effector function.

Outlook: It is becoming increasingly clear that T cell function is intimately linked to metabolic programs, and as such there is a considerable and growing interest in developing techniques that target metabolism for immunotherapy. Studying metabolism has often been difficult for the nonexpert, because many of the experimental approaches require specialized instrumentation that has not been widely available. Furthermore, acquiring sufficient cellular material for ex vivo analyses, coupled with the inherent difficulty of assessing cellular metabolism in vivo during an immune response, presents substantial challenges to scientists studying the metabolism of immune cells. Nevertheless, understanding how environmental cues and cellular metabolism influence the outcome of $\mathrm{T}$ cell-mediated immune responses will be critical for learning how to exploit metabolism to alter disease outcome. Overall, we are just beginning to understand the pathways that regulate metabolism in lymphocytes and how T cells adapt to changes in their microenvironment, particularly in vivo; this area of immunology is poised for substantial advances in the years to come.

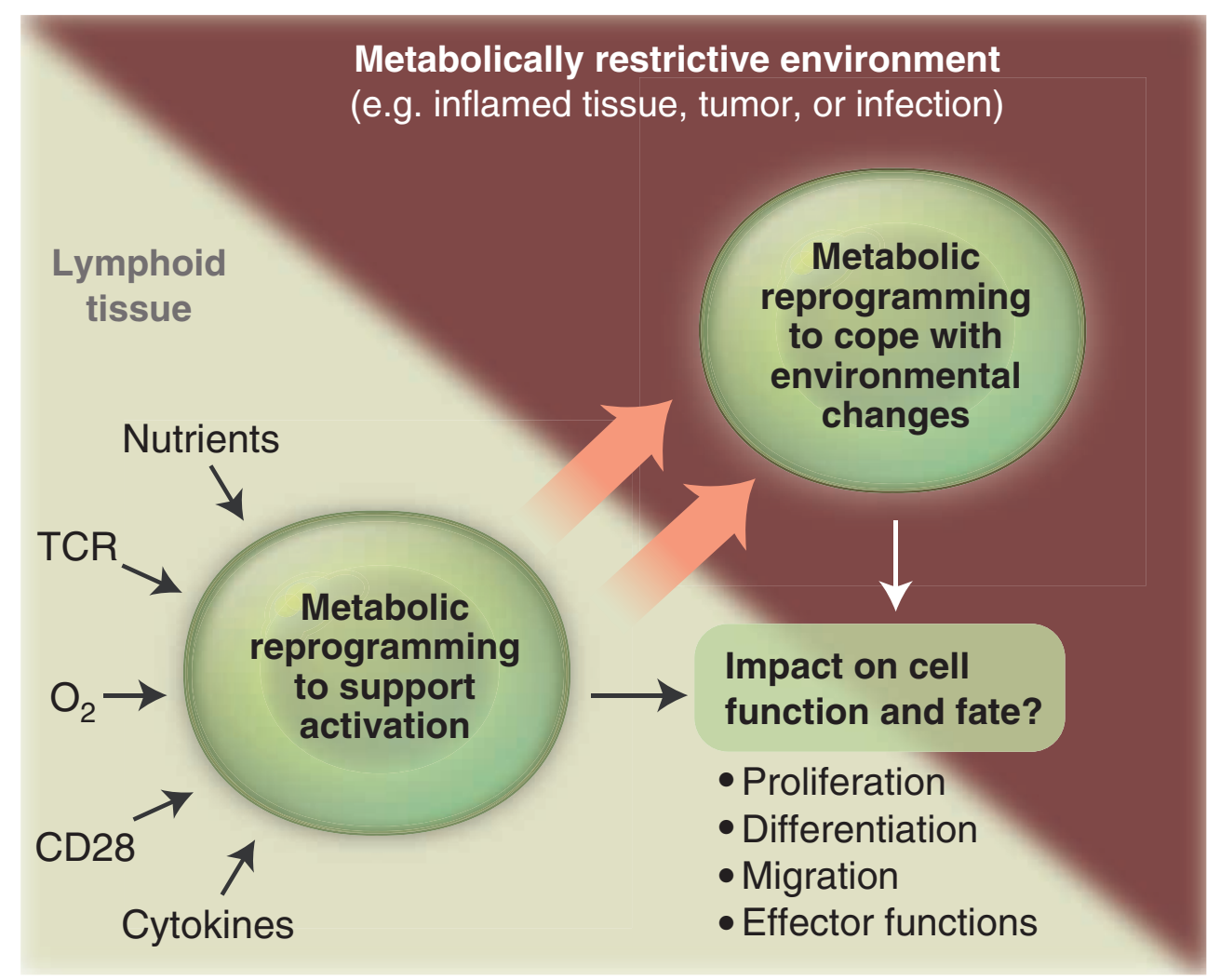

READ THE FULL ARTICLE ONLINE http://dx.doi.org/10.1126/science.1242454

Cite this article as Pearce et al. Science 342, 1242454 (2013) DOI: $10.1126 /$ science. 1242454

\section{ARTICLE OUTLINE}

Differential Regulation of T Cell Metabolism

Metabolism of Proliferating Cells: Lessons from Tumor Metabolism

Metabolites As Signaling Molecules

Future Challenges and Other Considerations

Concluding Thoughts

T cell function and fate are dependent on metabolic reprogramming. As T cells differentiate during an immune response, they move from what are presumably nutrient-replete lymphoid organs to sites of cancer or infection, where oxygen, nutrients, growth factors, and other signals may become limiting. These metabolically restrictive environments force T cells to metabolically adapt in order to survive and perform their necessary functions. 


\section{Fueling Immunity: Insights into Metabolism and Lymphocyte Function}

\author{
Erika L. Pearce, ${ }^{1_{*}}$ Maya C. Poffenberger, ${ }^{2,3}$ Chih-Hao Chang, ${ }^{1}$ Russell G. Jones ${ }^{2,3 *}$
}

Lymphocytes face major metabolic challenges upon activation. They must meet the bioenergetic and biosynthetic demands of increased cell proliferation and also adapt to changing environmental conditions, in which nutrients and oxygen may be limiting. An emerging theme in immunology is that metabolic reprogramming and lymphocyte activation are intricately linked. However, why T cells adopt specific metabolic programs and the impact that these programs have on $\mathrm{T}$ cell function and, ultimately, immunological outcome remain unclear. Research on tumor cell metabolism has provided valuable insight into metabolic pathways important for cell proliferation and the influence of metabolites themselves on signal transduction and epigenetic programming. In this Review, we highlight emerging concepts regarding metabolic reprogramming in proliferating cells and discuss their potential impact on T cell fate and function.

$\mathrm{T}$ The immune system is comprised of a series of specialized cells conditioned to respond rapidly to "danger" signals such as foreign pathogens or inflammatory stimuli. T lymphocytes, or $\mathrm{T}$ cells, are sentinels of the adaptive immune system that respond to antigen-specific signals by blasting, proliferating, and differentiating into effector subsets tailored to identify and eliminate threats to the host. Integrated into this program of activation is the regulation of cellular metabolism. Upon activation, $\mathrm{T}$ cells dramatically alter their metabolic activity to meet the increased metabolic demands of cell growth, proliferation, and effector function. Metabolism fundamentally underpins T cell function; thus, there is great interest in understanding how metabolic pathways influence immune responses and ultimately affect disease progression. It should be noted that "metabolism" refers to a complex network of biochemical reactions involved in energy production and macromolecular biosynthesis, and comprehensive coverage of such a broad topic is difficult. Several recent reviews have highlighted the molecular mechanisms that govern metabolic reprogramming in the immune system (1-3). This Review will focus on emerging areas in intermediary metabolism in lymphocytes and will discuss their potential impact on $\mathrm{T}$ cell fate, plasticity, and effector function.

\section{Differential Regulation of T Cell Metabolism}

\section{Lymphocyte Metabolism Is Dynamically Regulated}

Maintenance of cellular bioenergetics is an essential function of all living cells, and lymphocytes are no exception. In T lymphocytes, glucose is

${ }^{1}$ Department of Pathology and Immunology, Washington University School of Medicine, St. Louis, MO 63110, USA. ${ }^{2}$ Goodman Cancer Research Centre, McGill University, Montreal, QC H3G 1Y6, Canada. ${ }^{3}$ Department of Physiology, McGill University, Montreal, QC H3G 1Y6, Canada.

*Corresponding author. E-mail: erikapearce@path.wustl.edu (E.L.P.); russell.jones@mcgill.ca (R.G.J.) reinfection. a critical substrate for adenosine triphosphate (ATP) production (4). During glycolysis, glucose is broken down into two molecules of pyruvate. This process, which does not require oxygen, yields two reduced nicotinamide adenine dinucleotide (NADH) molecules and two net ATP molecules per molecule of glucose. Pyruvate has two alternate fates. Most terminally differentiated, nonproliferating cells can fully oxidize pyruvate in the tricarboxylic acid (TCA) cycle. This process generates NADH and reduced flavin adenine dinucleotide $\left(\mathrm{FADH}_{2}\right)$, which the cell can use to fuel OXPHOS, an oxygen-dependent process that produces up to 36 molecules of ATP per glucose molecule. Alternatively, pyruvate can be transformed (or fermented) into lactate, regenerating $\mathrm{NAD}^{+}$for subsequent use in glycolysis (5). From a bioenergetic perspective, engaging OXPHOS maximizes the amount of ATP that can be derived from glucose.

Bioenergetic profiling of $\mathrm{T}$ cells has revealed that $\mathrm{T}$ cell metabolism changes dynamically with activation state (Fig. 1). Upon antigen encounter, T cells become activated, undergo extensive proliferation, and differentiate into effector $\mathrm{T}$ cells $\left(\mathrm{T}_{\mathrm{EFF}}\right)$; upon pathogen clearance, most $\mathrm{T}_{\mathrm{EFF}}$ cells die, leaving behind a small population of long-lived antigen-specific memory $T$ cells $\left(\mathrm{T}_{\mathrm{M}}\right)$. Consistent with the metabolism of other nonproliferating cells, resting naïve $T$ cells ( $T$ cells that have not yet encountered antigen) maintain low rates of glycolysis and predominantly oxidize glucose-derived pyruvate via OXPHOS or engage fatty acid oxidation (FAO) to make ATP. Upon activation, T cells switch to a program of anabolic growth and biomass accumulation to generate daughter cells,

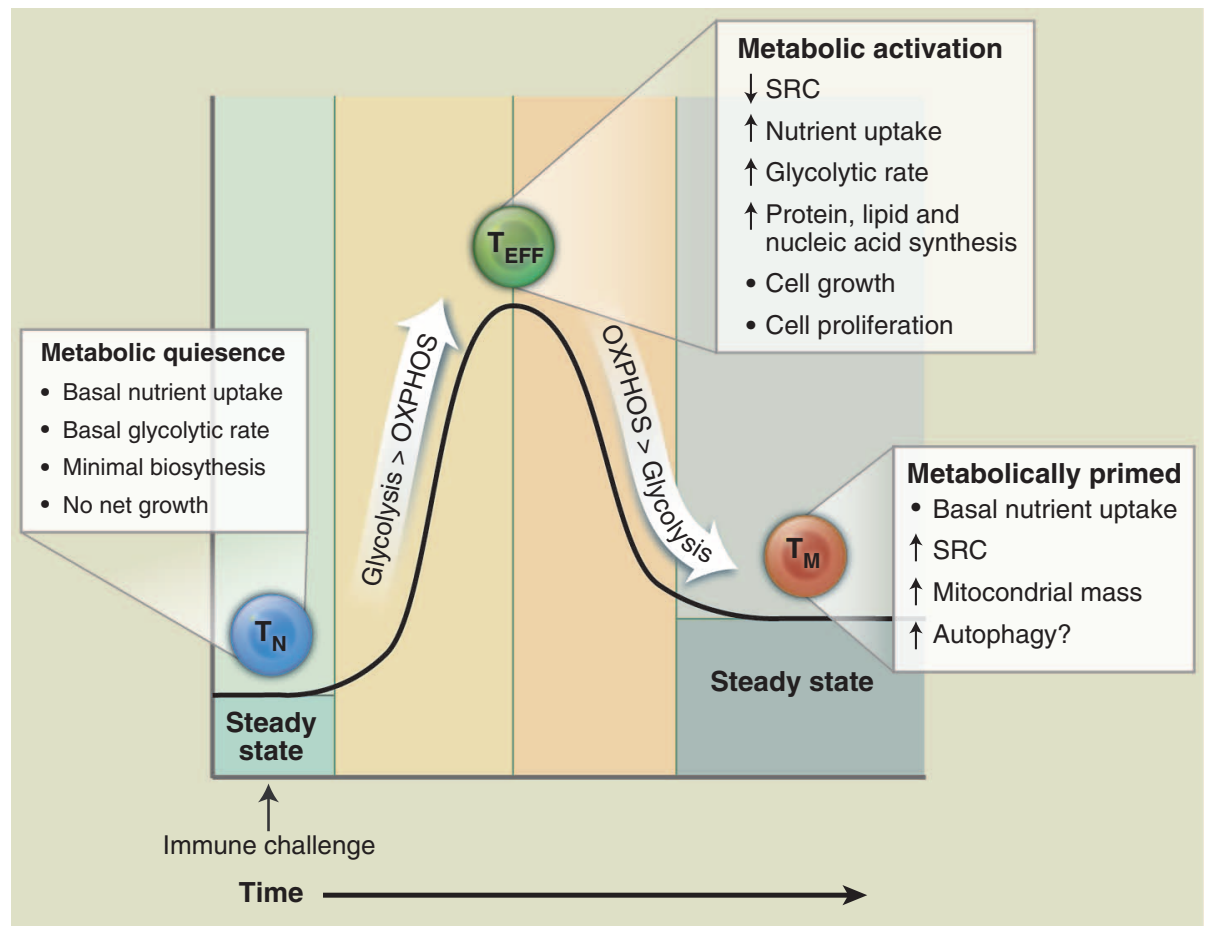

Fig. 1. T cell metabolism changes over the course of an immune response. $T$ cells display distinct metabolic profiles depending on their state of activation. Naïve $T$ cells $\left(T_{N}\right.$, blue) are metabolically quiescent; they adopt a basal level of nutrient uptake and use OXPHOS as their primary pathway of ATP production. Upon immune challenge, $T_{E F F}$ (green) cells shift to a state of metabolic activation characterized by increased nutrient uptake, elevated glycolytic and glutaminolytic metabolism, biomass accumulation, and reduced mitochondrial SRC. $T_{\text {EFF }}$ cells preferentially use glycolysis over OXPHOS for ATP production. Transition to the $T_{M}$ (orange) stage is characterized by a quiescent metabolism, with increased reliance on FAO to fuel OXPHOS. Mitochondrial mass and SRC are elevated in $T_{M}$ cells, suggesting that these cells are metabolically primed to respond upon 
which by definition dictates increased demand for ATP and metabolic resources. In this state, $T$ cells are considered to be metabolically activated (Fig. 1). T cell receptor (TCR) signaling directs the metabolic reprogramming of naïve $\mathrm{T}$ cells. TCR ligation promotes the coordinated up-regulation of glucose and amino acid transporters (6-8), facilitating nutrient uptake and T cell blastogenesis. TCR-mediated up-regulation of the transcription factors c-Myc (9) and estrogenrelated receptor $\alpha(E R R \alpha)(10)$ enhances the expression of genes involved in intermediary metabolism. In addition, catabolic pathways of ATP generation such as fatty acid $\beta$-oxidation are actively suppressed (9). The predominant metabolic phenotype of activated T cells is a shift to aerobic glycolysis [reviewed in (11)]. Both $\mathrm{CD}^{+}$ and $\mathrm{CD}^{+} \mathrm{T}_{\mathrm{EFF}}$ cells engage aerobic glycolysis, which is marked by the conversion of glucosederived pyruvate to lactate despite the availability of oxygen for complete glucose oxidation. This process, also known as the Warburg effect from earlier work in cancer biology, is a common trait of actively proliferating cells (5). It is important to note that OXPHOS is still engaged in $\mathrm{T}_{\mathrm{EFF}}$ cells (9); however, the production of lactate from pyruvate by aerobic glycolysis is the dominant pathway of glucose metabolism in $\mathrm{T}_{\mathrm{EFF}}$ cells. Regulating energy metabolism may provide a way for $T$ cells to reversibly switch between quiescent and highly proliferative states (12).

As a quiescent $T$ cell population, $T_{M}$ cells adopt a metabolic profile similar to that of naïve $\mathrm{T}$ cells - a catabolic metabolism characterized by increased reliance on OXPHOS and lower rates of nutrient uptake and biosynthesis relative to $\mathrm{T}_{\mathrm{EFF}}$ cells (Fig. 1). However, $\mathrm{T}_{\mathrm{M}}$ cells also display a characteristic increase in mitochondrial mass, which translates into greater mitochondrial spare respiratory capacity (SRC) relative to naïve or $\mathrm{T}_{\mathrm{EFF}}$ populations (13). SRC can be viewed as the maximal respiratory capacity available to a cell, much like the maximum speed that can be achieved by a car engine. Under increased workload, stress, or nutrient limitation, cells engage this reserve capacity to generate more energy and promote cell viability $(14,15)$. We have recently shown that increased mitochondrial mass and SRC of $\mathrm{T}_{\mathrm{M}}$ cells allows for rapid mitochondrial ATP production upon TCR engagement, conferring a bioenergetic advantage to $T_{M}$ cells upon secondary exposure to antigen (16). From this vantage, $T_{M}$ cells may be viewed as being metabolically primed, with mitochondrial metabolism fueling the rapid recall response to reinfection. The memory T cell-promoting cytokine interleukin (IL)-15 plays a key role in this catabolic switch by promoting mitochondrial biogenesis (13).

The mechanisms governing the transition of $\mathrm{T}$ cells from effector to memory states are still poorly understood, but recent work hints that changes in metabolism may influence this process. We previously demonstrated that mitochondrial FAO stimulated downstream of TNF (tumor necrosis factor) receptor-associated factor 6 (TRAF6) is required for memory $\mathrm{CD} 8^{+} \mathrm{T}$ cell development (17). Oxidation of free fatty acids (FFAs) generates acetyl-coenzyme A (CoA), which can be metabolized further in the TCA cycle, as well as $\mathrm{FADH}_{2}$ and NADH, which can be used directly by the electron transport chain (ETC) to make ATP. FFAs are energy-dense molecules, and FAO may be a preferred fuel source for $\mathrm{T}_{\mathrm{M}}$ cells as they rely on OXPHOS-dependent metabolic program. Administration of metformin, a metabolic stressor that activates the energy sensor adenosine monophosphate-activated protein kinase (AMPK), enhances the generation of $\mathrm{CD}^{+} \mathrm{T}$ cell memory (17). One consequence of AMPK activation is the suppression of mammalian target of rapamycin complex 1 (mTORC1) activity in response to energetic stress (18). Consistent with this, the drug rapamycin, which also inhibits $\mathrm{mTORC1}$, enhances the generation of $\mathrm{CD}^{+} \mathrm{T}_{\mathrm{M}}$ cells $(17,19,20)$. These observations suggest that manipulating the metabolism of antigen-specific cells during contraction can influence the development of $\mathrm{T}_{\mathrm{M}}$ cells. Given these observations, $T_{M}$ formation may be influenced by a number of enzymes and transporters involved in fatty acid synthesis, desaturation, and oxidation, as well as the availability of FFAs to memory precursor cells. Some important players to consider in this regard include acetylCoA carboxylase (ACC2) (21), the mitochondrial lipid transporter CPT1A $(13,22)$, and metabolites such as acetyl-CoA and malonyl-CoA (23). AMPK activation and mTOR inhibition are also both potent activators of autophagy, a catabolic process induced during starvation that promotes the degradation and recycling of cellular components [reviewed in detail in (24)]. Proper induction of autophagy has been shown to be important for the maintenance of cellular bioenergetics and sustained $\mathrm{T}$ cell viability after activation $(25,26)$. It will be interesting to determine whether autophagy, by coupling catabolic fuel supply to mitochondrial metabolism, is important for $\mathrm{T}_{\mathrm{M}}$ formation after infection.

\section{Mitochondrial OXPHOS and T Cell Activation}

Although much focus has been placed on the shift toward glycolysis that accompanies T cell activation, evidence suggests that mitochondrial OXPHOS is also important for $\mathrm{T}$ cell activation. Oligomycin, a specific inhibitor of mitochondrial ATP synthase, can block the expression of early activation markers after TCR ligation and blunts subsequent $\mathrm{T}$ cell proliferation (27), suggesting that the naïve-to-effector transition requires either de novo production of ATP by mitochondria or specific signals generated during mitochondrial ATP production. Mitochondrial-derived reactive oxygen species (ROS) may function as such a "bioenergetic" second messenger. There has long been evidence that ROS can play critical roles in shaping T cell responses (28-30). However, recent work suggests that mitochondrial ROS produced during OXPHOS is essential for T cell activation. $\mathrm{T}$ cells deficient for ubiquinol-cytochrome $\mathrm{c}$ reductase (Uqcrfs1), a component of complex III of the ETC, display impaired TCR-dependent ROS production and defects in antigen-specific proliferation (31). Intracellular calcium $\left(\mathrm{Ca}^{2+}\right)$ flux, an early event in TCR signal transduction, may provide the functional link between TCR ligation, mitochondrial OXPHOS, and cell proliferation. Uptake of $\mathrm{Ca}^{2+}$ by mitochondria stimulates $\mathrm{Ca}^{2+}$-dependent dehydrogenases of the TCA cycle, driving mitochondrial NADH production and ATP production by OXPHOS during early $\mathrm{T}$ cell activation (32). T cells lacking the apoptosis regulators Bax and Bak, which display defects in intracellular $\mathrm{Ca}^{2+}$ homeostasis, exhibit reduced $\mathrm{Ca}^{2+}$-dependent mitochondrial ROS production and $\mathrm{T}$ cell proliferation after TCR stimulation (33). Restoring $\mathrm{Ca}^{2+}$ signals in Bax/Bak-null T cells restores mitochondrial ROS production and $\mathrm{T}$ cell proliferation (33). Thus, although toxic in many biological settings, mitochondrial-dependent ROS may prime $\mathrm{T}$ cells and license full $\mathrm{T}$ cell activation.

\section{Metabolic Signatures Vary with Differentiation State}

Although the paradigm of T cell metabolism as summarized in Fig. 1 holds true with respect to activated versus quiescent states, the metabolic signature of T cells can also vary depending on differentiation state. This was first demonstrated by Michalek et al. (34), who determined that proinflammatory $C D 4^{+} \mathrm{T}$ helper $\left(\mathrm{T}_{\mathrm{H}}\right)$ cells $\left(\mathrm{T}_{\mathrm{H}} 1\right.$, $\mathrm{T}_{\mathrm{H}} 2$, and $\mathrm{T}_{\mathrm{H}} 17$ lineages) displayed a strong bias toward glycolysis over mitochondrial metabolism, whereas induced $\mathrm{CD}^{+} \mathrm{T}$ regulatory $\left(\mathrm{T}_{\text {reg }}\right)$ lineage cells displayed a mixed metabolism involving glycolysis, lipid oxidation, and OXPHOS. In particular, $\mathrm{T}_{\mathrm{H}} 17$ cells display increased reliance on glycolysis for their development and maintenance. $\mathrm{T}_{\mathrm{H}} 17$ cell development is promoted by hypoxia inducible factor- $1 \alpha(\mathrm{HIF}-1 \alpha)(35,36)$, an oxygen-sensitive transcription factor that regulates glycolytic gene expression in $\mathrm{T}_{\mathrm{H}} 17$ cells. Blocking glycolysis during $\mathrm{T}_{\mathrm{H}} 17$ cell differentiation reduced the development of $\mathrm{T}_{\mathrm{H}} 17$ cells and favored the formation of $\mathrm{T}_{\text {regs }}(35)$. Added to this are recent results indicating that extracellular salt $(\mathrm{NaCl})(37,38)$ and short-chain fatty acids (39) can influence $T_{H} 17$ and $T_{\text {reg }}$ homeostasis, respectively. This raises the intriguing possibility that the metabolic microenvironment (i.e., nutrient and oxygen availability) can influence $\mathrm{T}$ cell polarization (to be discussed later). Determining whether the metabolic signature of differentiated T cells is simply a consequence of lineage-specific cytokine signaling or is instructive for T cell function (i.e., essential for regulating $\mathrm{T}$ cell plasticity and/or effector function) remains a question for the field. Examining the influence of key metabolic regulators such as HIF- $1 \alpha$, mTOR, and AMPK on T cell differentiation and plasticity will help in resolving these issues.

\section{Metabolism of Proliferating Cells: Lessons from Tumor Metabolism}

Research in cancer metabolism over the past 10 years has increased our understanding of the 
metabolic requirements of proliferating cells, as well as the metabolic alterations that promote tumor growth. One of the great innovations we have learned from the cancer metabolism field is that signaling pathways, or more specifically the oncogenes and tumor suppressors that comprise and regulate signal transduction pathways, can influence cellular metabolism as part of their program of action. The previous paradigm of metabolic regulation argued that metabolic pathways were exclusively controlled through allosteric regulation of metabolic enzymes, either by ATP levels or metabolites themselves (the reactive model) (40). One example of this is the glycolytic enzyme phosphofructokinase (PFK), which is inhibited allosterically by ATP and citrate (indicating a high energy state in the cell) and stimulated by AMP (indicative of low energy). Although allosteric regulation is important for regulating local flux through metabolic pathways, we now understand that activation of signal transduction pathways (such as phosphatidylinositol 3-kinase or Akt) by growth factor receptors stimulates global changes in metabolic flux independent of ATP levels. This allows a cell that receives a proliferative signal, such as a $\mathrm{T}$ cell activated through its TCR, to drive cellular metabolism above the capacity normally maintained in the quiescent state. Overall, metabolism in T cells is likely regulated at several levels: (i) TCRmediated changes in the expression of metabolic genes facilitate the reprogramming required to match metabolic pathways to biological need; (ii) signal transduction downstream of cell surface receptors (i.e., costimulatory molecules and cytokine receptors) serve to fine-tune flux through these metabolic pathways; and (iii) feedback inhibition and other forms of allosteric regulation can regulate metabolic flux through local nodes in the network. By directly influencing metabolic reprogramming, oncogenes and tumor suppressors gain control over the metabolic currency of the cell, namely, energetic intermediates $\left(\mathrm{ATP}, \mathrm{NAD}^{+} / \mathrm{NADH}, \mathrm{FAD}^{+} / \mathrm{FADH}_{2}\right.$, and $\left.\mathrm{NADP}^{+} / \mathrm{NADPH}\right)$ and metabolites involved in bioenergetic and biosynthetic reactions that influence cell growth and survival.

The implication of these findings for immunologists is that metabolic pathways are indirectly connected to cell surface receptors of the immune system via signal transduction pathways. TCR/CD28 stimulation of T cells ( 6 ), the stimulation of surface immunoglobulin on B cells (41), and TLR stimulation of macrophages and dendritic cells (DCs) $(42,43)$ all promote changes in aerobic glycolysis characteristic of the Warburg effect. These results likely just scratch the surface of the complex metabolic networks at work in proliferating cells. The challenge going forward will be to identify key pathways of metabolic flux integral for lymphocyte function. In this regard, research into tumor cell metabolism has provided valuable insight into the metabolic pathways important for cell proliferation. Many of the metabolic pathways abnormally activated in cancer, such as aerobic glycolysis, have been shown to play similar roles in normal lymphocyte physiology. Here, we highlight recent advances in intermediate metabolism observed in cancer that are likely to be relevant to $\mathrm{T}$ cell biology.

\section{The Warburg Effect: More than ATP Synthesis}

Rapid glucose processing promoted by the Warburg effect allows proliferating T cells to generate ATP quickly; glycolysis also generates metabolic intermediates important for cell growth and proliferation (Fig. 2). Metabolism of glucose through the oxidative or nonoxidative arms of the pentose phosphate pathway (PPP) generates ribose5-phosphate (Rib-5P), a key intermediate in nucleotide biosynthesis. The oxidative arm of the PPP also produces NADPH, the key metabolic currency for nucleotide and fatty acid biosynthesis. T cell activation promotes a rapid increase in glucose flux through the oxidative PPP (9). Dihydroxyacetone-phosphate (DHAP) is used to generate the glycerol backbone for glycerophospholipids, and 3-phosphoglycerate (3PG) is a key intermediate in both amino acid and nucleotide biosynthesis (discussed below). Pyruvate that is not converted to lactate can enter into the mitochondria and be converted into acetyl-CoA by the pyruvate dehydrogenase (PDH) complex. In proliferating cells, mitochondria adopt an additional role as a biosynthetic hub, converting pyruvate and other metabolites into metabolic intermediates involved in protein and fatty acid biosynthesis (44).

One of the key metabolic intermediates for biosynthesis is acetyl-CoA. Acetyl-CoA has a central role in membrane biogenesis because it provides two-carbon units for fatty acid and isoprenoid biosynthesis, as well as in other diverse processes such as protein prenylation and $\mathrm{N}$ glycosylation (45). The flow of glucose to the cytosolic acetyl-CoA pool is regulated by using TCA cycle intermediates and a truncated TCA cycle (46). In this model, mitochondrial citrate is formed from condensation of oxaloacetate and acetyl-CoA, after which citrate is exported from the mitochondrion to the cytosol and converted back to acetyl-CoA by ATP citrate lyase (ACL) (47). Despite the availability of extracellular lipids for membrane biosynthesis, FFAs are generated de novo from glucose in proliferating cells using this pathway (46). CD8+ T cells unable to engage acetyl-CoA-dependent lipid biosynthetic pathways display defects in antigendriven blastogenesis and clonal expansion in response to pathogens (48). Acetyl-CoA can also influence metabolic flux through acetylation of metabolic enzymes $(49,50)$, reinforcing metabolic pathways such as glycolysis when carbon availability is high. Glucose availability and acetylCoA production can also influence epigenetics by regulating the cytosolic acetyl-CoA pools available for histone acetylation reactions (51), raising the prospect that glucose-dependent metabolic flux may help drive or reinforce T cell differentiation programs.
One consequence of using glucose-derived mitochondrial citrate for lipid biosynthesis is the potential depletion of TCA cycle intermediates. Oxaloacetate (OAA) is a rate-limiting substrate for acetyl-CoA entry into the TCA cycle. OAA generated from ACL-mediated cleavage of cytosolic citrate can potentially cycle back into the mitochondria to maintain the TCA cycle. Inefficient cycling of this pathway would lead to cumulative depletion of mitochondrial OAA, leading to collapse of the TCA cycle and disruption of mitochondrial function. One way tumor cells counter this is by engaging glutaminolysis, a metabolic shunt that converts glutamine into $\alpha$-ketoglutarate $(\alpha-K G$, also known as 2-oxoglutarate) for use in the TCA cycle (Fig. 2). Glutamine has long been known as a key metabolite for supporting $\mathrm{T}$ cell function (52). Recent evidence suggests that glutamine metabolism is as dynamically regulated in T cells as glucose metabolism. Glutamine transporters (SNAT1 and SNAT2) as well as key glutaminolysis enzymes (GLS, GPT, GOT, and GLUD) are upregulated early after $\mathrm{T}$ cell activation similar to glycolysis genes $(7,9)$; several groups have correlated these changes in gene expression to enhanced glutaminolytic flux in lymphocytes $(9,53)$. Recently, it was found that engagement of the TCR leads to the expression of Slc7a5, an amino acid transporter that mediates the import of large neutral amino acids, such as leucine (8). Amino acid influx via this transporter is required for the activation of mTOR and expression of c-Myc and as such coordinates activation-induced metabolic reprogramming and differentiation of $\mathrm{T}$ cells. Thus, amino acids such as glutamine and leucine appear to play additional roles in T cell function beyond protein biosynthesis and may directly influence $\mathrm{T}$ cell activation by regulating metabolic reprogramming.

\section{Control of Glycolytic Flux by Pyruvate Kinase M2 (PKM2)}

Pyruvate kinase (PK) is a key enzyme of the glycolytic pathway. It catalyzes the terminal reaction of glycolysis by promoting the conversion of phosphoenolpyruvate (PEP) to pyruvate (Fig. 2), and is one of two ATP-generating steps of glycolysis (the second is mediated by phosphoglycerate kinase). The muscle version of PK exists as one of two isoforms, M1 or M2, generated from differential splicing of the PKM primary mRNA transcript, with the PKM2 splice variant expressed in embryonic tissues, proliferating cells, and tumor cells (54). Naïve T cells express both M1 and M2 isoforms at rest; mitogen-dependent activation promotes the rapid accumulation of the M2 isoform, which becomes the dominant isoform expressed in $\mathrm{T}_{\mathrm{EFF}}$ cells (55). PKM2 exists as either an inactive dimer or an active tetramer, and oscillation between these two states influences the ability of cells to maintain anabolic metabolism (56).

Multiple lines of evidence point to a role for PKM2 in coordinating glycolytic flux and cell 


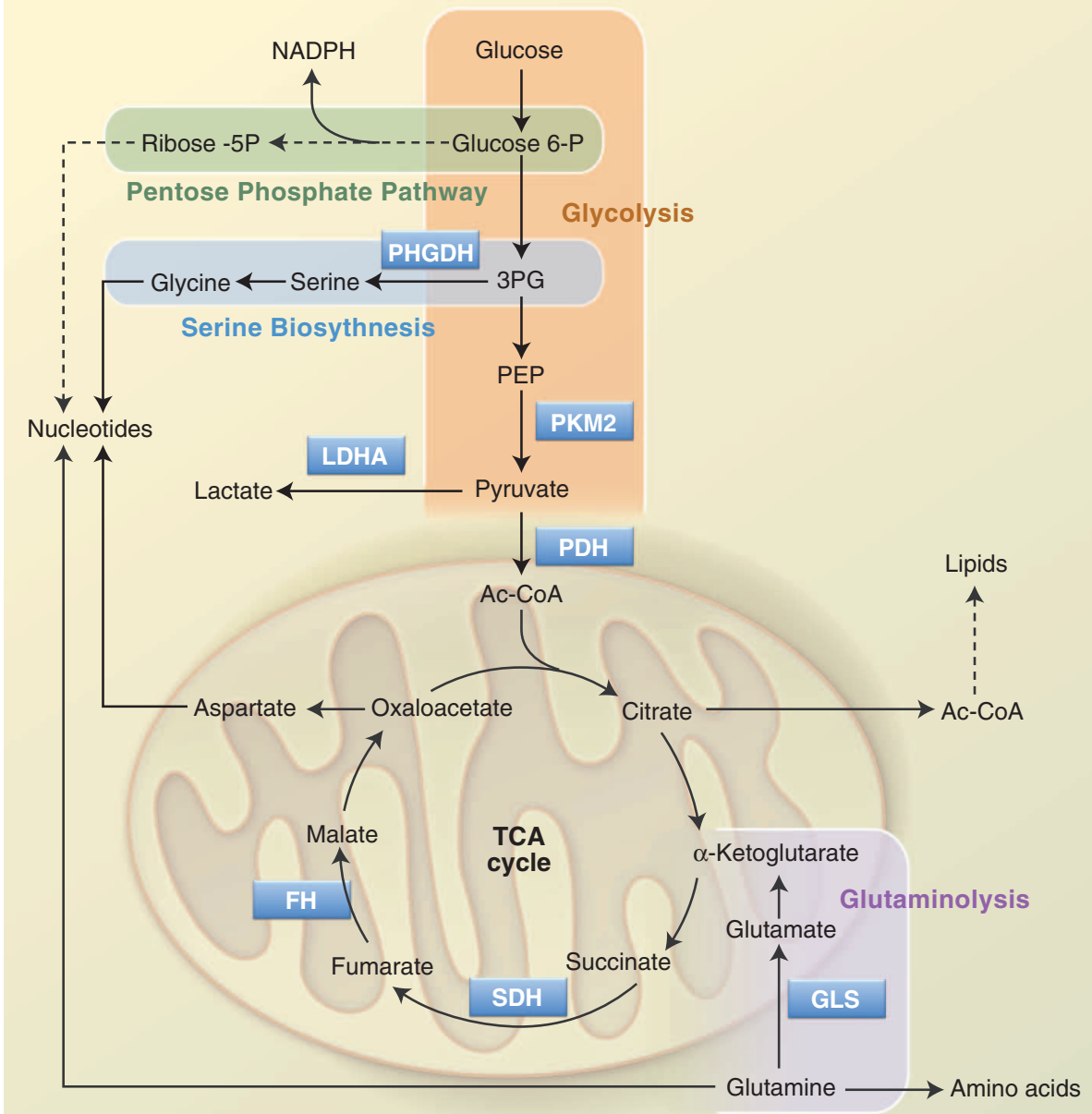

Fig. 2. Metabolic pathways that support cell growth and proliferation. Glycolysis and the TCA cycle are two separate yet connected biochemical pathways that function to generate ATP as well as metabolic precursors for biosynthesis. Glucose is broken down to pyruvate by glycolysis (orange); pyruvate can be further oxidized by the TCA cycle in the mitochondrion. Glycolytic intermediates can be used to generate other metabolites required for growth and proliferation. Glucose 6-phosphate and 3PG produced from glycolysis are metabolized in the PPP (green) and the SBP (blue), respectively, providing important precursors for nucleotide biosynthesis. Similarly, acetyl-CoA, generated from glucose-derived citrate in the TCA cycle, can be used for lipid biosynthesis. OAA, produced as part of the TCA cycle, can be used to generate aspartate, another precursor for nucleotide synthesis. An alternate source of carbon for the TCA cycle occurs via glutaminolysis (purple); in this pathway, glutamine is converted to glutamate and then to $\alpha-K G$, which joins the TCA cycle. Glutamine is also a precursor for amino acid and nucleotide biosynthesis. Key enzymes in these pathways are PHGDH; PKM2; LDHA, lactate dehydrogenase; PDH; GLS, glutaminase; SDH, succinate dehydrogenase; and FH.

proliferation. Tumor cells engineered to exclusively express the M2 isoform display increased lactate production characteristic of the Warburg effect and gain a growth advantage in vivo over M1expressing tumors (57). Interestingly, the M2 isoform is actually less efficient at converting PEP to pyruvate than the M1 isoform. Moreover, growthfactor-stimulated tyrosine phosphorylation of PKM2 further decreases its activity $(58,59)$. This prompts the question: Why would proliferating cells, including T cells, promote the expression of a pyruvate kinase isoform that is less efficient at generating ATP? The answer to this question may lie with the second function of Warburg metabolism, namely, supporting anabolic growth. Buildup of PEP because of reduced PKM2 ac- nologists is that PKM2 can phosphorylate signal transducer and activator of transcription 3 (STAT3) at $\mathrm{Tyr}^{705}$, promoting increased STAT3-dependent transcription (63). The phosphorylation of STAT3 by PKM2 demonstrates that PKM2 possesses both protein and pyruvate kinase activities, the former using PEP as a phosphate donor rather than ATP. The protein kinase activity of PKM2 (and any subsequent effects on STAT3 activity) would be predicted to be sensitive to metabolic flux, favored under high-glycolysis PEP conditions and antagonized by low-energy high-ADP concentrations. The development of mouse models to study the impact of PKM2 activity in vivo will be important for elucidating the role(s) of PKM2 in immune function.

\section{The Serine Biosynthesis Pathway}

Another glycolytic intermediate that can double as an anabolic precursor is 3PG. 3PG is the starting point for the glucose-dependent biosynthesis of serine and glycine via the serine biosynthesis pathway (SBP) (Fig. 3A). Key enzymes of the SBP are phosphoglycerate dehydrogenase (PHGDH), the rate-limiting step for serine biosynthesis, and serine hydroxymethyltransferase (SHMT), which uses serine as a methyl donor to convert tetrahydrofolate (THF) to methylene-THF, generating glycine in the process. Methylene-THF is a key intermediate in folate-mediated one-carbon metabolism that fuels nucleotide biosynthesis and methylation reactions. Serine is also an allosteric activator of PKM2 (64) and thus provides feedback to the glycolytic pathway to regulate $3 \mathrm{PG}$ levels and serine biosynthesis.

In mammals, serine and glycine are nonessential amino acids and are widely abundant in serum (and tissue culture medium). However, glucose-dependent serine biosynthesis is actively engaged in some tumors regardless of serine abundance. Amplification of $P H G D H$ has been observed in breast cancer and melanoma (65), and increased PHGDH expression can promote both enhanced serine biosynthesis and the proliferation of cancer cells $(65,66)$. Enhanced flux through the SBP may confer a growth advantage to tumor cells beyond providing increased serine and glycine for biosynthetic reactions. First, PHGDH produces NADH, which can be used to maintain cytosolic redox balance or fuel mitochondrial OXPHOS to make ATP. The second step of the pathway, the conversion of 3-phosphohydroxypyruvate to 3-phosphoserine by phosphoserine aminotransferase (PSAT), requires glutamate and produces $\alpha-\mathrm{KG}$ (Fig. 3A) (66). Thus, the SBP may promote an alternate pathway of $\alpha-K G$ production for mitochondrial metabolism or promote the activity of $\alpha-\mathrm{KG}$ dependent enzymes (to be discussed later). Last, serine and glycine are both intermediates in the production of reduced glutathione (GSH), a key cellular antioxidant. Recent evidence indicates that cancer cells actively produce GSH from glucose via the SBP as a buffer against oxidative damage (67). The expression of SBP enzymes is 


\section{A}
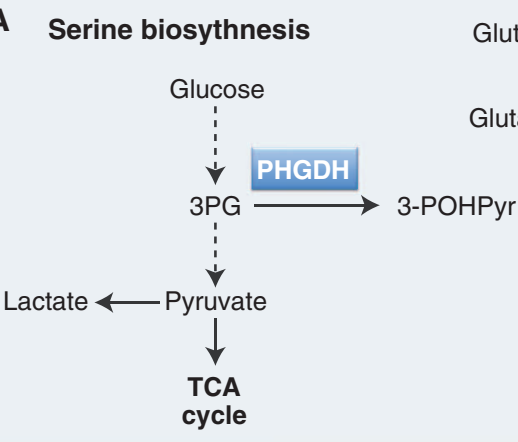

Glutamine
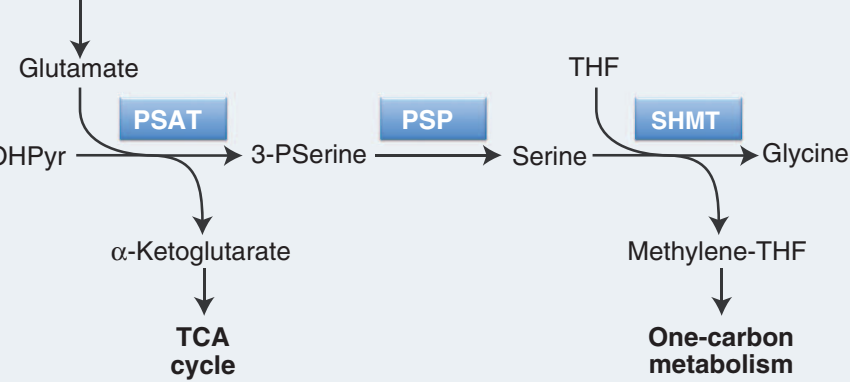

B

Normal
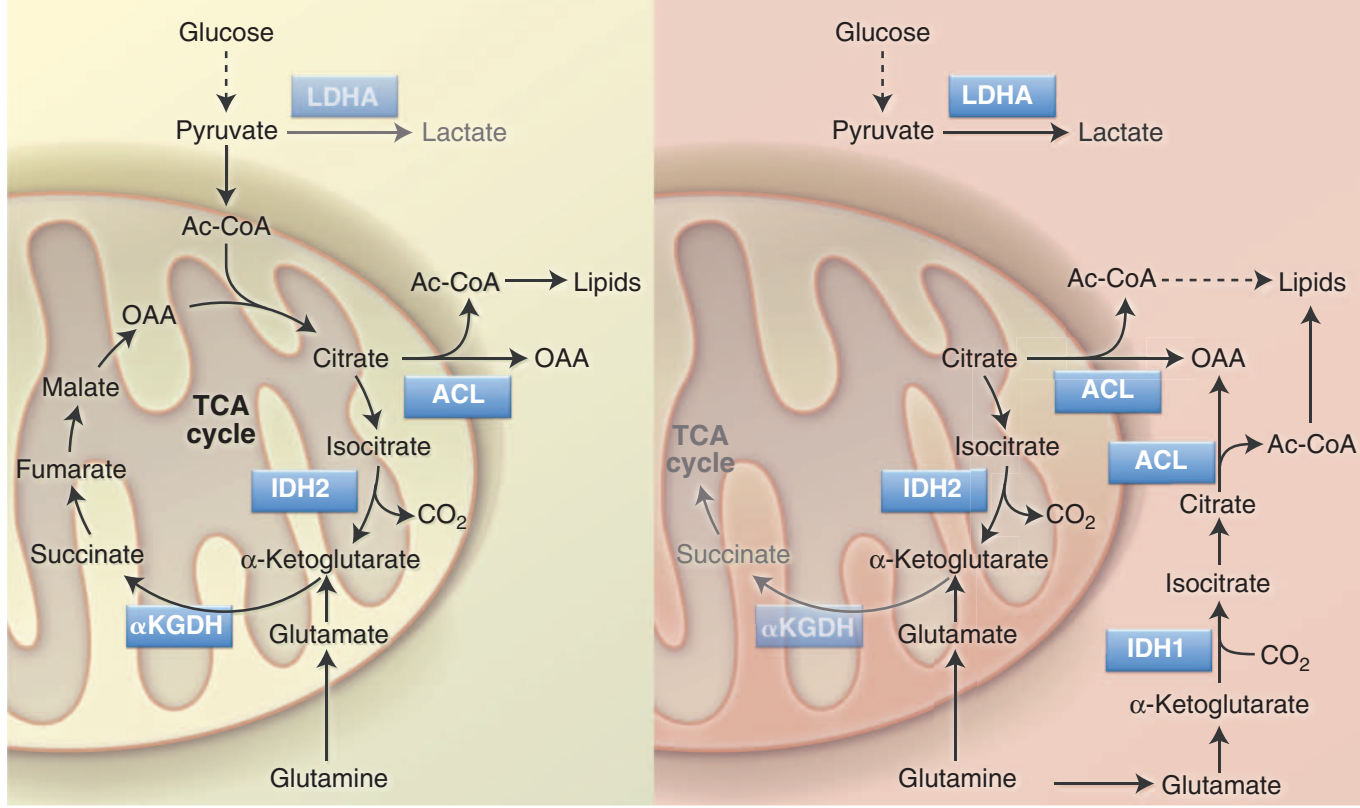

Fig. 3. Serine biosynthesis and reductive carboxylation are anabolic pathways that support cell proliferation.

(A) The SBP converts glucose-derived 3PG into serine and glycine, which are precursors for lipid and nucleotide biosynthesis. Serine is also involved in folate-mediated one carbon metabolism by acting as a methyl group donor for THF to methylene-THF conversion. Key enzymes in this pathway are PHGDH, PSAT, and SHMT. (B) Reductive carboxylation is an alternate pathway of glutamine metabolism in which glutamine-derived $\alpha$-KG is converted to citrate through reverse TCA cycle flux. Under conditions of hypoxia or mitochondrial dysfunction (right), isocitrate dehydrogenase (IDH1 in cytosol, $\mathrm{IDH} 2$ in mitochondria) uses $\mathrm{CO}_{2}$ and NADPH to convert $\alpha$-KG into isocitrate. Citrate produced downstream of this reaction is converted into cytosolic acetyl-CoA without passing through the conventional clockwise steps of the TCA cycle. Acetyl$\mathrm{CoA}$ generated by this pathway can function as a precursor for fatty acid synthesis. $\alpha-K G D H, \alpha-K G$ dehydrogenase.

such as hypoxia or mitochondrial dysfunction (70-72). In this reaction, glutamine-derived $\alpha-K G$ is carboxylated, rather than decarboxylated, by IDH1 (in the cytosol) or IDH2 (in mitochondria) to generate citrate (Fig. 3B). This switch to reductive versus oxidative metabolism of $\alpha-\mathrm{KG}$ is regulated in part by $\operatorname{HIF}-1 \alpha(71,72)$, although the exact mechanism by which HIF-1 $\alpha$ does so remains unclear. Engaging reductive carboxylation of $\alpha-K G$ in essence bypasses the conventional TCA cycle by using glutamine to generate the acetyl-CoA required for fatty acid synthesis (Fig. 3B). Under such metabolic reprogramming, cancer cells continue to use glycolysis for ATP production but switch from glucose to glutamine as the major lipogenic precursor.

One implication of this work is that tumor cells display a high degree of metabolic plasticity and can adapt their metabolism to support proliferation and viability under fluctuating environmental conditions. Whether T cells display similar metabolic flexibility in response to environmental cues requires further investigation. The work from Metallo and colleagues (71) suggests that $\mathrm{T}$ cells can use reductive glutamine metabolism for fatty acid biosynthesis under hypoxic conditions. Physiologic oxygen tension varies significantly between tissues, and hypoxic regions can be detected in the bone marrow, thymus, and spleen (73). Moreover, T cells are likely to experience hypoxia at sites of tissue inflammation (74). Stabilization of HIF- $1 \alpha$ and metabolic reprogramming to favor reductive carboxylation may help $\mathrm{T}$ cells maintain proliferation and/or effector function at hypoxic inflammatory sites. HIF-1 $\alpha$

up-regulated in Myc-driven lymphomas (68); thus, we hypothesize that $T_{\mathrm{EFF}}$ cells may actively engage the SBP upon activation, even in the presence of exogenous serine. Future investigation will be needed to determine how the SBP influences T cell biology.

\section{Reductive Carboxylation of $\alpha-K G$}

As mentioned previously, it is now appreciated that in proliferating cells the TCA cycle functions as a source of biosynthetic precursors in addition to its role in ATP production (46). Carbon enters the TCA cycle primarily at one of two entry points: (i) the condensation of glucose-derived acetylCoA with OAA to generate citrate and (ii) conversion of glutamine to $\alpha-\mathrm{KG}$ via glutaminolysis (Fig. 3B). Carbon-tracing experiments using pro- liferating glioblastoma cells have established that both glucose and glutamine contribute to mitochondrial citrate pools and subsequent lipid synthesis (69). The oxidative decarboxylation of isocitrate to $\alpha-\mathrm{KG}$ by isocitrate dehydrogenase (IDH) is highly favored thermodynamically, such that this reaction is believed to be irreversible and the reason for the "clockwise" flow of metabolic intermediates through the TCA cycle.

Groundbreaking new work suggests that metabolite flow through biochemical pathways does not always conform to conventional dogma. Although oxidation of glutamine-derived $\alpha-\mathrm{KG}$ in the TCA cycle serves as a minor source of lipogenic acetyl-CoA under normal growth conditions, $\alpha-K G$ can be converted to citrate through reductive carboxylation under conditions of stress protein expression has also been observed early after T cell activation (75), so it is unclear whether reductive carboxylation may also be engaged as part of the normal program of $\mathrm{T}$ cell expansion. As mentioned, HIF- $1 \alpha$ has been implicated in the differentiation of both proinflammatory $\mathrm{T}_{\mathrm{H}} 17$ cells $(35,36)$ and $\mathrm{CD}^{+}{ }^{+}$FoxP3 $^{+} \mathrm{T}_{\text {reg }}$ cells $(76)$. It is tempting to speculate that this alternate pathway of glutamine metabolism may influence the expansion or phenotypic stability of these T cell subsets.

\section{Metabolites As Signaling Molecules}

Cancer genome sequencing efforts yielded an unexpected discovery in 2008 with the identification of somatic mutations in a metabolic enzyme, the TCA cycle enzyme IDH1, in glioblastoma 
multiforme (GBM) (77). Mutations in IDH1 presented at significant frequency $(\sim 12 \%$ of GBM patients) with high frequency of missense mutations targeting an arginine residue in the active enzyme site $\left(\operatorname{Arg}^{132}\right)$. This mutation alters the enzymatic ability of IDH1, allowing it to convert $\alpha-K G$ to 2 -hydroxyglutarate $(2-\mathrm{HG})$ rather than promote normal isocitrate-to- $\alpha-\mathrm{KG}$ interconversion (78). In the cancer setting, 2-HG has been shown to influence a number of biological processes, including cell differentiation, DNA methylation, and histone methylation (79-82), leading to its classification as an oncometabolite. These results have shifted thinking in cancer biology to consider that specific metabolites may also act as signaling molecules to influence cell physiology. Given certain similarities between metabolic programming in tumor cells and proliferating T cells, it stands to reason that metabolites will participate in $\mathrm{T}$ cell signaling as well. Some examples of how metabolites shape T cell function and fate through metabolic pathways are discussed in detail here.

\section{Regulation of LKB1-AMPK Signaling by Adenylates}

ATP is the primary carrier of chemical energy in the cell and essential for life. Thus, adenylates [ATP, adeonsine diphosphate (ADP), and AMP] are important units of cellular metabolic currency. In mammalian cells, fluctuations in cellular energy are monitored by the heterotrimeric AMPK complex [reviewed in (83)]. ATP, ADP, and AMP compete for nucleotide-binding sites of the $\gamma$ regulatory subunit of AMPK, with AMP (low energy) promoting and ATP (high energy) antagonizing AMPK activation. As such, AMPK functions as a sensor of the cellular adenylate energy charge $(84,85)$. Elevation of the cellular AMP:ATP ratio leads to increased phosphorylation of AMPK at $\mathrm{Thr}^{172}$ of its activation loop by the kinase LKB1 (Fig. 4A). AMPK can also be activated by $\mathrm{Ca}^{2+}$ (via CamKK $\beta$ ) and the cytokine transforming growth factor $-\beta$ (TGF- $\beta$ ) (via TAK1), although LKB1 appears to be the sole kinase that couples adenylate binding to AMPK activation.

Together LKB1 and AMPK function as part of an evolutionarily conserved energy-sensing pathway that maintains cellular energy balance by promoting catabolic pathways of ATP production and limiting processes that consume ATP. Protein synthesis is one of the most energyconsuming processes in the cell, accounting for $\sim 20 \%$ of cellular ATP consumption (86). As mentioned, AMPK antagonizes mRNA translation through negative regulation of mTORC1 (Fig. 4A) (18). By regulating AMPK activity, adenylates directly influence pathways of energy usage in the cell. As ATP levels drop, AMP binds to AMPK, and AMPK is switched on to promote ATP production and block its consumption; once ATP homeostasis has been reestablished, increased binding of ATP to AMPK shuts off the kinase.

Recent work indicates that LKB1-AMPK signaling can influence $\mathrm{T}$ cell metabolism and function. Lymphocytes exclusively express the $\alpha 1$ catalytic subunit of AMPK (87), and TCR stimulation promotes LKB1-dependent AMPK activation in lymphocytes $(87,88)$. Glycolysis is enhanced in resting AMPK $\alpha$-deficient T cells (88), consistent with observations that silencing AMPK in tumor cells promotes the Warburg effect (89). Loss of LKB1-AMPK signaling promotes increased mTORC1 activity in both naïve and $\mathrm{T}_{\mathrm{EFF}}$ cells $(88,90)$, which in turn facilitates production of the $\mathrm{T}_{\mathrm{H}} 1$ cytokine interferon- $\gamma(\mathrm{IFN}-\gamma)$ by $\mathrm{T}_{\mathrm{EFF}}$ cells (88). Thus, LKB1 and AMPK act in concert to limit the anabolic growth of $\mathrm{T}$ cells by suppressing glycolysis and mTOR-dependent biosynthesis. Perhaps not surprisingly, deletion of either LKB1 or AMPK $\alpha 1$ disrupts normal lym- phocyte homeostasis, resulting in an accumulation of activated $\left(\mathrm{CD} 44^{\text {hi }} \mathrm{CD} 2 \mathrm{~L}^{\text {lo }}\right) \mathrm{CD}^{+} \mathrm{T}$ cells in animals (88). These results suggest that cellular adenylate levels and AMPK may help regulate lymphocyte pools in the whole organism.

Why would a signaling pathway that normally monitors cellular energy levels regulate T cell function? Similar to tumor cells (91), AMPK may regulate a metabolic checkpoint in T cells, acting as a brake on lymphocyte expansion when energy conditions are poor. $\mathrm{T}_{\mathrm{EFF}}$ cells with defective AMPK signaling would be freed from these metabolic checkpoints and continue to proliferate and produce cytokines as if cellular bioenergetics were suitable to support $\mathrm{T}$ cell function. Additionally, AMPK may regulate the metabolic plasticity of lymphocytes, coordinating metabolic changes in response to nutrient fluctuation and allowing T cells to survive changes in their environment. As evidence for this, the AMPK agonist metformin, which promotes FAO in activated $\mathrm{T}$ cells, enhances the production of $\mathrm{CD} 8^{+}$ memory T cells in vivo (17). Furthermore, it was recently shown that AMPK-deficient $\mathrm{T}$ cells are defective in their ability to generate $\mathrm{CD} 8^{+}$memory $\mathrm{T}$ cells during infection (92). There is also a growing body of evidence implicating AMPK in the control of inflammation (3). Future work will focus on the role of AMPK in regulating the metabolic fitness of lymphocytes, dissecting LKB1and AMPK-specific effects on immune function and investigating the role(s) of LKB1 and AMPK in regulating inflammation in vivo.

\section{Regulation of $\alpha$-Ketoglutarate-Dependent Enzymes by TCA Cycle Metabolites}

Although TCA cycle metabolites play central roles in energy metabolism, many function as chemical intermediates in other biological reactions. For example, fumarate can be used to chemically modify cysteine residues of proteins, resulting

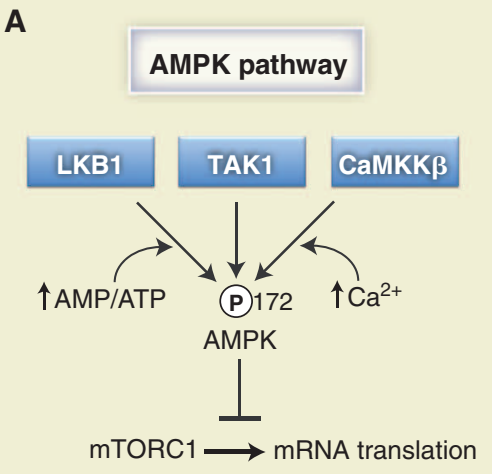

B

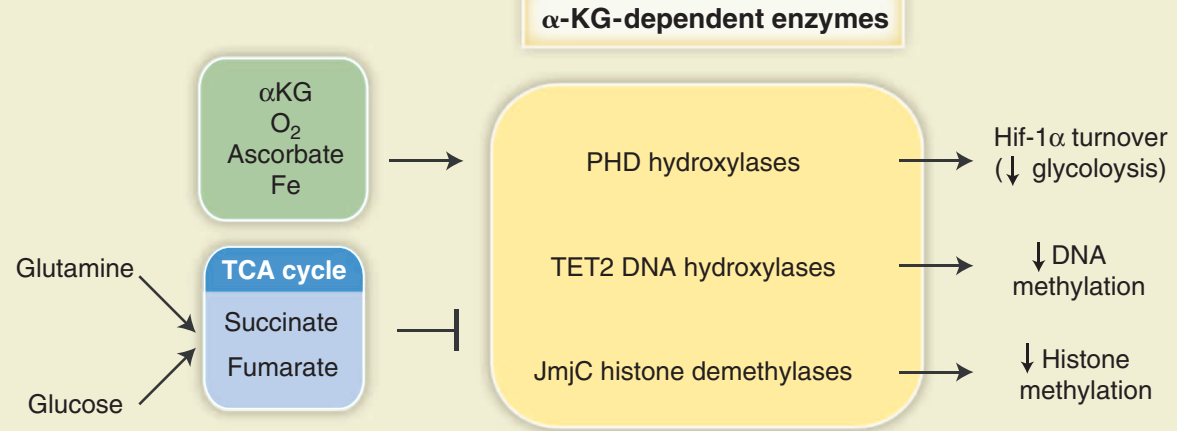

Fig. 4. Metabolites can influence signal transduction. (A) The AMPK pathway is influenced by adenylate concentration. AMPK $\alpha$ is activated by phosphorylation on Thr $^{172}$ of its activation loop by the kinases LKB1, TAK1, or CaMKK $\beta$. LKB1 promotes enhanced AMPK phosphorylation under a high AMP:ATP ratio. One biological output of AMPK activity is the inhibition of mRNA translation under low-energy conditions through inhibition of mTORC1 activity. (B) $\alpha-K G-$ dependent enzymes (in yellow) are a class of enzymes regulated by TCA cycle intermediates that require molecular oxygen $\left(\mathrm{O}_{2}\right)$ and $\alpha$-KG for their enzymatic activity. Oxygen, $\alpha-K G$, ascorbate, and iron (green) are positive regulators of these enzymes, whereas the TCA cycle intermediates fumarate and succinate (blue) antagonize their reactions. PHDs destabilize HIF-1 $\alpha$ protein, resulting in decreased expression of HIF- $1 \alpha$ targets and a reduction in glycolysis. TET2 hydroxylates 5-methylcytosine residues to promote DNA demethylation, whereas ]mjC promotes demethylation of trimethylated histones in chromatin. 
in the formation of S-(2-succinyl)cysteine or succinylation of these residues (93). Cancer cells harboring mutations in fumarate hydratase $(\mathrm{FH})$, the TCA cycle enzyme that converts fumarate to malate, accumulate intracellular fumarate and display increased amounts of succinylated proteins (94). Increased protein succinylation has been associated with renal carcinoma and mechanistically can influence signal transduction pathways similar to protein phosphorylation or acetylation (95).

$\alpha-\mathrm{KG}$ is another key metabolite involved in non-TCA cycle biochemical reactions. $\alpha-K G$ is essential for the activity of a class of enzymes known as $\alpha-\mathrm{KG}-$ dependent dioxygenases (Fig. 4B). These enzymes use $\alpha-K G$ along with molecular $\mathrm{O}_{2}$, iron, and ascorbate to modify target proteins (96). The most extensively characterized enzymes of this family are the HIF prolyl hydroxylases (PHDs). PHDs use $\mathrm{O}_{2}$ to hydroxylate HIF-1 $\alpha$ on two conserved proline residues, facilitating their recognition by the E3 ubiquitin ligase VHL and promoting HIF- $1 \alpha$ degradation by the proteosome. When $\mathrm{O}_{2}$ availability is low, HIF-1 $\alpha$ protein is stabilized because of reduced PHD activity, resulting in increased HIF-1 $\alpha-$ dependent transcription (97). This regulatory circuit allows HIF-1 $\alpha$ to promote glycolytic ATP production when $\mathrm{O}_{2}$ cannot support mitochondrial OXPHOS, an example of metabolic adaptation in response to environmental conditions. Other $\alpha-K G$-dependent enzymes using this chemistry are the TET family of DNA hydroxylases, which hydroxylate 5-methylcytosine residues to promote DNA demethylation, and the Jumonji C (JmjC) class of histone demethylases (Fig. 4B). A detailed summary of these processes has recently been reviewed (98).

The activity of $\alpha-\mathrm{KG}$-dependent enzymes is directly affected by the abundance of TCA cycle intermediates. For example, 2-HG acts as a competitive inhibitor of $\alpha-K G$, and its production by mutant IDH1 consumes $\alpha-\mathrm{KG}(81,99)$, leading to reduced TET2 and JmjC activity in tumor cells $(79,80)$. High levels of succinate and fumarate can inhibit PHD2 activity through productmediated inhibition of PHD function, leading to HIF-1 $\alpha$ protein stabilization under normoxic $\left(20 \% \mathrm{O}_{2}\right)$ conditions $(100,101)$. Additionally, total abundance of $\alpha-\mathrm{KG}$ is low in most cell types. The implication of these findings is that metabolic flux through the TCA cycle can affect gene transcription and/or epigenetic programs. It was recently shown that succinate plays a central role in production of the cytokine IL- $1 \beta$ by lipopolysaccharide (LPS)-stimulated macrophages (102). LPS induces the de novo production of succinate from glutamine, leading to PHD inhibition, stabilization of HIF-1 $\alpha$, and increased HIF- $1 \alpha$-dependent IL- $1 \beta$ production. Mutant $\mathrm{IDH} 2\left[\mathrm{Arg}^{140} \rightarrow \mathrm{Gln}^{140}\right.$ (R140Q)], which promotes $\alpha-K G$ depletion/2-HG production, promotes DNA hypermethylation in hematopoietic cells and can inhibit myeloid differentiation when overexpressed in bone marrow stem cells (79). Differentiation of $\mathrm{T}$ cells to specific $\mathrm{T}_{\mathrm{H}}$ lineages is driven by spe-

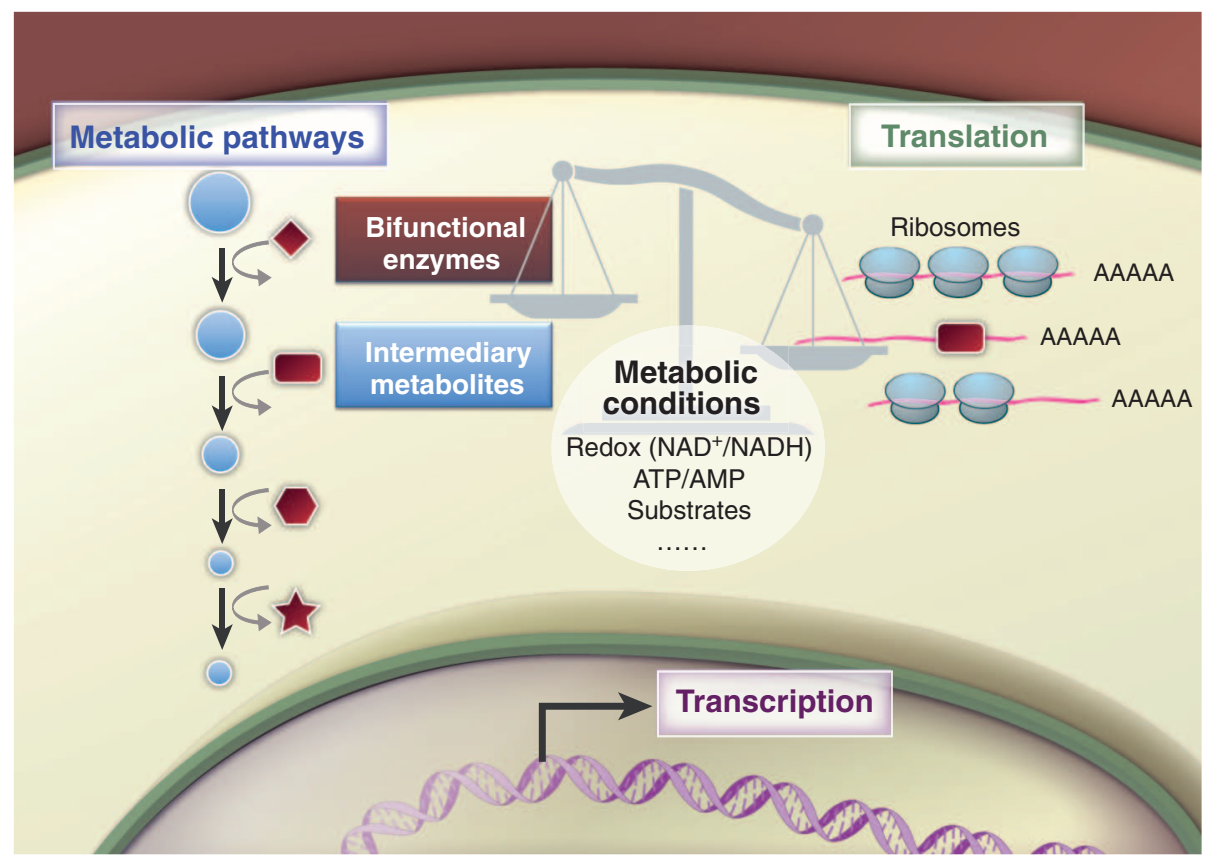

Fig. 5. Bifunctional metabolic enzymes connect metabolism and gene regulation. Metabolic enzymes can moonlight as RNA binding proteins and regulate the translation of specific target mRNAs. The RNA binding function of enzymes can be influenced by interactions with intermediary metabolites and cofactors, leading to posttranscriptional regulation of protein expression. Posttranslational modification of metabolic enzymes could influence their RNA binding function directly or by altering the enzyme's subcellular location. Changes in metabolic conditions, such as bioenergetic demand, hypoxia, stress, and substrate availability, may affect the consequences of the REM interactions. The overall balance of the network between RNA, enzymes, and metabolites can potentially influence T cell fate and function.

cific transcription factors but reinforced by epigenetic modifications on histone tails (H3K4 and H3K27 trimethylation) and DNA methylation of CpG dinucleotides (103). Differential TCA cycle flux or buildup of intermediates such as succinate or fumarate may influence the activity of $\alpha-\mathrm{KG}-$ dependent enzymes that regulate $\mathrm{T}$ cell epigenetics. This may be one way in which the environment $\left(\mathrm{O}_{2}\right.$, nutrient levels) can influence the plasticity of $\mathrm{T}_{\mathrm{H}}$ lineages at sites of infection or inflammation in vivo. Thus, much like the role of oncometabolites in tumorigenesis, studying the metabolism of $\mathrm{T}$ cell responses may reveal the existence of "immunometabolites" that influence T cell responses and inflammation.

\section{Connecting Metabolism and Gene Regulation}

A transformative conceptual change in the way we consider metabolism within cells is that changes in metabolism can be linked to changes in gene expression through posttranscriptional regulatory networks involving RNA, metabolites, and metabolic enzymes "moonlighting" as RNA binding proteins and regulating specific target mRNAs (104) (Fig. 5). Many enzymes, including those connected with the TCA cycle, glycolysis, PPP, fatty acid metabolism, and other pathways, have been shown to bind RNA in vitro and in cultured cells $(105,106)$. In addition, the RNA binding function of enzymes can be influenced by interactions with their metabolites or cofactors, illus- trating how the metabolic state of the cell can control an enzyme's RNA binding function.

The metabolic enzyme perhaps best characterized for its physiological role as an RNA binding protein is cytosolic aconitase $(107,108)$, a key regulator of cellular iron metabolism (109). In the early 1990s, it was shown that cytosolic aconitase and the RNA binding protein IRP-1 represent the same polypeptide and that the availability of iron triggers insertion or removal of an iron sulfur cluster - switching the protein's function between RNA binding activity (iron low, IRP-1) and metabolic enzyme activity (iron high, aconitase) (110). Remarkably, IRP-1 binds to and regulates mRNAs encoding proteins that function in iron homeostasis. Work from this group and others has led to the idea that bifunctional enzymes and RNA binding proteins may represent a general mechanism of how metabolism and gene expression are coordinated through RNA/enzyme/metabolite (REM) networks [proposed by Hentze and Preiss in (104)].

Lending weight to the REM network hypothesis is a recent study showing that the enzyme glyceraldehyde-3-phosphate dehydrogenase (GAPDH), by engaging or disengaging the glycolysis pathway and through fluctuations in its expression, regulates the posttranscriptional production of IFN- $\gamma$ by T cells (27). GAPDH is a multifunctional enzyme that can bind a range of RNAs, including AU-rich regions in the 3' 
untranslated region (UTR) of cytokine mRNAs, including IFN- $\gamma$ and IL-2 (111). Activated T cells can use either OXPHOS or glycolysis to generate energy to support proliferation and survival. When T cells switch between these ATPgenerating programs, as can occur with changes in nutrient availability, or costimulatory or growth factor signals, GAPDH switches from its function as a metabolic enzyme (glycolysis) to its function as an RNA binding protein controlling expression of immunomodulatory factors. When GAPDH is not engaged in glycolysis and OXPHOS is used for ATP production, it binds the 3'UTR of cytokine mRNAs, and translation of these mRNAs is dampened. Thus although OXPHOS can support $T$ cell survival and proliferation, only aerobic glycolysis can facilitate full effector status. This regulatory mechanism provides a checkpoint to allow for uncoupling of $\mathrm{T}$ cell proliferation and survival from cytokine production. This is a desirable control mechanism over effector function, because $\mathrm{T}$ cells are required to undergo both homeostatic proliferation, when IFN- $\gamma$ production is neither required nor desirable, and antigendriven proliferation during an immune response, when effector cytokine production is essential.

The activity of GAPDH is not only controlled by pathway engagement as dictated by substrate availability but is also heavily influenced by redox balance within the cell. For example, GAPDH requires $\mathrm{NAD}^{+}$for its enzymatic function, but $\mathrm{NAD}^{+}$also interferes with mRNA binding, at least in vitro (112). Thus, $\mathrm{NAD}^{+}$controls both enzyme activity and RNA binding in a mutually exclusive fashion. Metabolic enzymes that regulate $\mathrm{NAD}^{+} / \mathrm{NADH}$ balance, including LDHA and $\mathrm{PHGDH}$, may also influence this process. Redox changes may also affect posttranslational modifications of GAPDH, altering its binding to mRNA, metabolites, as well as its localization within the cell $(113,114)$. This level of regulation between redox balance and RNA binding would be expected to occur with other RNA binding enzymes that share similar dinucleotide binding motifs with GAPDH. Although much work needs to be done to fully understand the biological importance of the interactions between enzymes, RNA, and metabolites, these observations clearly demonstrate how cofactors and substrates generally considered for their direct effects on metabolism may also coordinate metabolism with gene expression.

\section{Future Challenges and Other Considerations}

\section{Technical Challenges in Studying Lymphocyte Metabolism}

Although interest in studying lymphocyte metabolism and technological advances in metabolomics have increased over the past several years, the field faces many challenges going forward. For instance, although equipment for studying basic metabolism (i.e., oxygen consumption and proton production) is becoming more commonplace, specialized equipment for metabolite measure- ments [i.e., mass spectrometry (MS), nuclear magnetic resonance (NMR) spectroscopy] and analytical expertise is not routinely accessible to investigators. Another limitation is that, unlike microarray or sequencing technologies, metabolomic analyses do not adhere to a single global platform. For example, gas chromatography coupled to MS (GC-MS) is effective at quantifying organic acids such as TCA cycle intermediates but not most glycolytic intermediates. Thus, multiple platforms must be used to generate complete metabolite data sets. Current extraction methods do not allow for the measurement of subcellular metabolite pools, so information on metabolite localization or channeling between organelles is also lost. One caution of measuring steady-state metabolite levels is that these data provide no measurement of metabolite flux, that is, the speed or direction of metabolite flow through a given pathway (115). Metabolic flux analysis using isotopically labeled metabolites such as ${ }^{13} \mathrm{C}$ glucose or ${ }^{13} \mathrm{C}$-glutamine will be essential for delineating pathways of metabolite flow in T cells.

Perhaps the most relevant challenge for immunologists is the amount of material required for metabolomic analysis. Because of advances in flow cytometry and the identification of new biomarkers to define $T$ cell subsets, immunology has entered an era of cellular subspecialization, where rare cell populations are readily characterized. In comparison, a typical metabolic flux experiment requires millions of cells. This raises the issue of having limited material to analyze the metabolism of cells grown in vitro, let alone in vivo. The development of instrumentation with increased sensitivity will help reduce this gap, but better sample preparation and techniques to amplify metabolite signals are badly needed. The development of genetically encoded fluorescent biosensors for metabolic activity, such as those recently developed for NADH (116) and cellular energy charge (117), will be particularly powerful for studying $\mathrm{T}$ cell metabolism at the singlecell level.

\section{Microenvironmental Effects on Metabolism: Are Our Model Systems Correct?}

One of the ongoing questions regarding current experimental models in immunology is how closely cell culture models recapitulate immune responses in vivo. From a metabolic perspective, one can conclude that the two systems are worlds apart. Activated T cells cultured in standard medium (Iscove's modified Dulbecco medium plus $10 \%$ serum) experience $25 \mathrm{mM}$ glucose (five times standard blood glucose of $5 \mathrm{mM}$ ), $4 \mathrm{mM}$ glutamine (eight times the standard concentration of $0.5 \mathrm{mM}$ in blood), and $20 \% \mathrm{O}_{2}$ [two to four times the oxygen tension in blood, which varies depending on tissue type (73)]. Most in vitro assays to assess $\mathrm{T}$ cell function are performed at nutrient and $\mathrm{O}_{2}$ levels much higher than seen in normal physiology; these conditions model a hyperglycemic and hyperoxic environment never seen in vivo.
As $\mathrm{T}_{\mathrm{EFF}}$ cells move from a nutrient-replete environment in the lymph node or spleen to distant sites of infection, they are likely to experience more restrictive metabolic environments (Fig. 6). Some tissue sites, such as the thymus, bone marrow, and the gastrointestinal epithelium, are naturally hypoxic, whereas inflammation can promote local hypoxic regions in tissues (73). Unlike $\mathrm{O}_{2}$, which can freely diffuse into tissues, nutrients such as glucose move through space by Brownian motion and require transport into cells, and thus they are likely to have a much more restricted distribution in tissues. Local metabolic activity of immune cells at the site of infection can rapidly consume available $\mathrm{O}_{2}$ and nutrients, resulting in metabolic stress for infiltrating $\mathrm{T}$ cell populations (Fig. 6). There is evidence that $\mathrm{T}_{\mathrm{EFF}}$ cells in the tumor microenvironment compete with tumor cells for available glucose, and this competition model of nutrient restriction limits the ability of $\mathrm{T}_{\mathrm{EFF}}$ cells to produce effector cytokines such as IFN- $\gamma$ (27). Thus, metabolic and environmental influences on $\mathrm{T}$ cell function in vivo may elicit very different responses and may account for experimental variance between $\mathrm{T}$ cell responses in a petri dish versus what is observed in animal models. Although these points provide sobering food for thought, the development of in vitro methods that control metabolic parameters (e.g., hypoxia incubators, perfusion systems for culture medium) may help to reconcile these differences. Studying metabolite flux of $\mathrm{T}$ cells in vivo by using isotopomer labeling techniques will further our understanding of metabolic pathways relevant for $\mathrm{T}$ cell function. These techniques are currently being developed in the cancer biology field $(118,119)$ and should be readily transferable to immunology research.

If activated $\mathrm{T}$ cells frequently transition between nutrient-replete states (lymph nodes) and nutrient-deficient states (sites of infection), then management of metabolic resources is an important consideration for lymphocytes in order to ensure maintenance of proliferation and/or effector function. Metabolic insufficiency may be a fundamental mechanism by which environmental context regulates $\mathrm{T}$ cell function, potentially influencing T cell tolerance and anergy. Metabolic interference mechanisms, such as indoleamine 2,3-dioxygenase (IDO)-1-dependent degradation of tryptophan by antigen presenting cells (APCs), may represent key regulatory mechanisms at sites of infection or inflammation (120). Tumors may similarly restrict antitumor immunity by influencing $\mathrm{T}$ cell metabolism. Competition between tumor cells and tumor-infiltrating $\mathrm{T}$ cells for available glucose can impose nutrient deprivation on $\mathrm{T}_{\mathrm{EFF}}$ cells that limits their ability to produce effector cytokines (27). Tumor-derived lactate can also suppress CTL function directly by blocking lactate export by $\mathrm{T}$ cells, thus disrupting their ability to maintain glycolysis (121).

It remains to be determined whether $\mathrm{T}$ cells deal with nutrient restriction by displaying metabolic plasticity similar to tumor cells. In this con- 


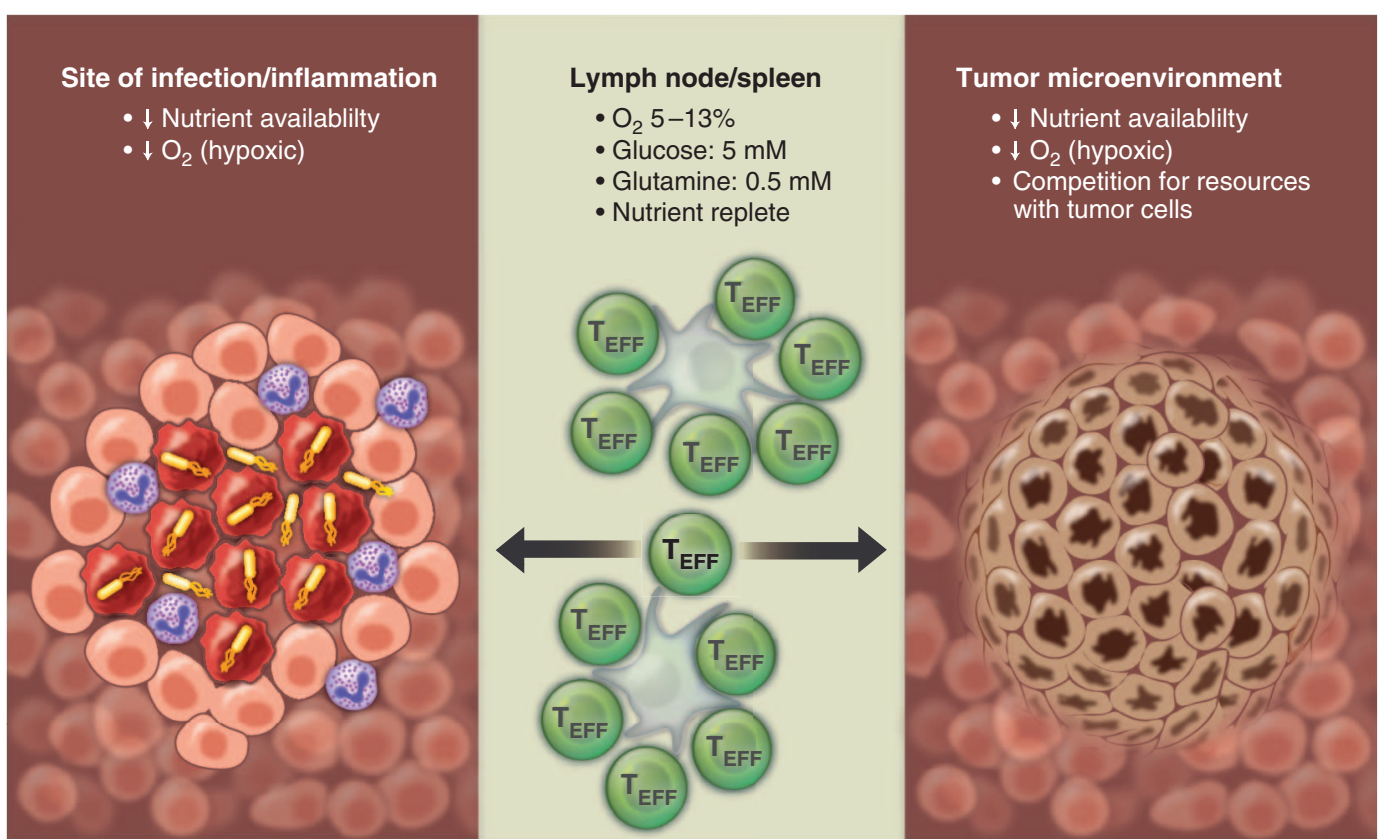

Fig. 6. T cells must display metabolic plasticity to adapt to changes in nutrient and oxygen availability in vivo. $\mathrm{T}_{\mathrm{EFF}}$ (green) cells must adapt to varying oxygen and nutrient levels depending on environmental context. Lymphoid organs (middle) are considered to be nutrient- and oxygen-replete areas, whereas sites of inflammation (left) and the tumor microenvironment (right) contain hypoxic areas with fluctuations in nutrient availability. At sites of inflammation, nutrient and oxygen availability may become limited because of the metabolic activity of cells at the site of inflammation, necrosis of infected cells, and oxygen consumption by neutrophils. Tumor microenvironments can be highly hypoxic resulting from insufficient vascularization. Additionally, T cells must compete with tumor cells for nutrients such as glucose.

doi: 10.4049/jimmunol.0903586; pmid: 20554958

8. L. V. Sinclair et al., Control of amino-acid transport by antigen receptors coordinates the metabolic reprogramming essential for $T$ cell differentiation. Nat. Immunol. 14, 500-508 (2013). doi: 10.1038/ ni.2556; pmid: 23525088

9. R. Wang et al., The transcription factor Myc controls metabolic reprogramming upon T lymphocyte activation. Immunity 35, 871-882 (2011). doi: 10.1016/j.immuni.2011. 09.021; pmid: 22195744

10. R. D. Michalek et al., Estrogen-related receptor- $\alpha$ is a metabolic regulator of effector T-cell activation and differentiation. Proc. Natl. Acad. Sci. U.S.A. 108, 18348-18353 (2011). doi: 10.1073/pnas.1108856108; pmid: 22042850

11. N. J. Maclver, R. D. Michalek, J. C. Rathmell, Metabolic regulation of T lymphocytes. Annu. Rev. Immunol. 31, 259-283 (2013). doi: 10.1146/ annurev-immunol-032712-095956; pmid: 23298210

12. E. L. Pearce, Metabolism in T cell activation and differentiation. Curr. Opin. Immunol. 22, 314-320 (2010) doi: 10.1016/j.coi.2010.01.018; pmid: 20189791

13. G. J. van der Windt et al., Mitochondrial respiratory capacity is a critical regulator of $\mathrm{CD} 8+\mathrm{T}$ cell memory development. Immunity 36, 68-78 (2012). doi: 10.1016/j.immuni.2011. 12.007; pmid: 22206904

text, AMPK and HIF-1 $\alpha$, which monitor cellular energy and $\mathrm{O}_{2}$ levels, respectively, are likely to have a profound effect on the adaptive immune response. Loss of HIF-1 $\alpha$ impairs $\mathrm{T}_{\mathrm{H}} 17$ expansion and induction of experimental autoimmune encephalitis (35), consistent with a requirement for $\mathrm{HIF}-1 \alpha$ in promoting $\mathrm{T}_{\mathrm{H}} 17$ function in vivo. Overall, we know very little regarding pathways that regulate metabolism and adaptation to metabolic stress in lymphocytes, particularly in vivo; this area of immunology is poised for important discoveries in the years to come.

\section{Concluding Thoughts}

Recent findings in metabolism and cancer biology have rapidly closed the gap between cell signaling and biochemical pathways. One can now consider all parameters of these fields as being directly intertwined, comprising an interconnected network from gene expression to metabolite production. T cells provide a unique opportunity to understand how metabolism is used in normal biology to achieve proliferation versus abnormal biology, such as that observed in cancer. Characterizing how these pathways are integrated in T cells, how perturbations in the system (i.e., nutrient availability, $\mathrm{O}_{2}$ tension, and metabolite flux) influence $\mathrm{T}$ cell responses, and how metabolic responses are regulated in vivo in the context of infection will be some of the challenges facing scientists in this field. Understanding how environmental cues and cellular metabolism influence the outcome of $\mathrm{T}$ cell- mediated immune responses will continue to be an active area of research in the future. Interfering with metabolic pathways by using agents such as metformin and rapamycin has already revealed substantial and unexpected effects on T cellmediated immunity. Understanding how metabolic reprogramming influences $\mathrm{T}$ cell fate and effector function has the potential to uncover new ways of modulating $\mathrm{T}$ cell responses.

\section{References and Notes}

1. R. Wang, D. R. Green, Metabolic checkpoints in activated T cells. Nat. Immunol. 13, 907-915 (2012). doi: 10.1038/ni.2386; pmid: 22990888

2. E. L. Pearce, E. J. Pearce, Metabolic pathways in immune cell activation and quiescence. Immunity 38, 633-643 (2013). doi: 10.1016/j.immuni.2013.04.005; pmid: 23601682

3. L. A. O'Neill, D. G. Hardie, Metabolism of inflammation limited by AMPK and pseudo-starvation. Nature $\mathbf{4 9 3}$ 346-355 (2013). doi: 10.1038/nature11862; pmid: 23325217

4. E. F. Greiner, M. Guppy, K. Brand, Glucose is essential for proliferation and the glycolytic enzyme induction that provokes a transition to glycolytic energy production. J. Biol. Chem. 269, 31484-31490 (1994). pmid: 7989314

5. M. G. Vander Heiden, L. C. Cantley, C. B. Thompson, Understanding the Warburg effect: The metabolic requirements of cell proliferation. Science 324, 1029-1033 (2009). doi: 10.1126/science.1160809. pmid: 19460998

6. K. A. Frauwirth et al., The CD28 signaling pathway regulates glucose metabolism. Immunity 16, 769-777 (2002). doi: 10.1016/S1074-7613(02)00323-0; pmid: 12121659

7. E. L. Carr et al., Glutamine uptake and metabolism are coordinately regulated by ERK/MAPK during T lymphocyte activation. J. Immunol. 185, 1037-1044 (2010).
14. N. Yadava, D. G. Nicholls, Spare respiratory capacity rather than oxidative stress regulates glutamate excitotoxicity after partial respiratory inhibition of mitochondrial complex I with rotenone. J. Neurosci. 27, 7310-7317 (2007). doi: 10.1523/JNEUROSCI.0212-07. 2007; pmid: 17611283

15. D. G. Nicholls, Spare respiratory capacity, oxidative stress and excitotoxicity. Biochem. Soc. Trans. 37, 1385-1388 (2009). doi: 10.1042/BST0371385; pmid: 19909281

16. G. J. van der Windt et al., CD8 memory T cells have a bioenergetic advantage that underlies their rapid recall ability. Proc. Natl. Acad. Sci. U.S.A. 110, 14336-14341 (2013). doi: 10.1073/pnas.1221740110; pmid: 23940348

17. E. L. Pearce et al., Enhancing CD8 T-cell memory by modulating fatty acid metabolism. Nature 460 , 103-107 (2009). doi: 10.1038/nature08097; pmid: 19494812

18. D. B. Shackelford, R. J. Shaw, The LKB1-AMPK pathway: Metabolism and growth control in tumour suppression. Nat. Rev. Cancer 9. 563-575 (2009). doi: 10.1038/ nrc2676; pmid: 19629071

19. R. R. Rao, Q. Li, K. Odunsi, P. A. Shrikant, The mTOR kinase determines effector versus memory CD8+ T cell fate by regulating the expression of transcription factors T-bet and Eomesodermin. Immunity 32, 67-78 (2010). doi: 10.1016/j.immuni.2009.10.010 pmid: 20060330

20. K. Araki et al., mTOR regulates memory CD8 T-cell differentiation. Nature 460, 108-112 (2009) doi: 10.1038/nature08155; pmid: 19543266

21. L. Abu-Elheiga, M. M. Matzuk, K. A. Abo-Hashema, S. ]. Wakil, Continuous fatty acid oxidation and reduced fat storage in mice lacking acetyl-CoA carboxylase 2 . Science 291, 2613-2616 (2001). doi: 10.1126/ science.1056843; pmid: 11283375

22. R. J. DeBerardinis, J. J. Lum, C. B. Thompson, Phosphatidylinositol 3-kinase-dependent modulation of carnitine palmitoyltransferase $1 \mathrm{~A}$ expression regulates lipid metabolism during hematopoietic cell growth. J. Biol. Chem. 281, 37372-37380 (2006). doi: 10.1074/ jbc.M608372200; pmid: 17030509 
23. J. D. McGarry, G. P. Mannaerts, D. W. Foster, A possible role for malonyl-COA in the regulation of hepatic fatty acid oxidation and ketogenesis. J. Clin. Invest. 60, 265-270 (1977). doi: 10.1172/]Cl108764; pmid: 874089

24. M. Kundu, C. B. Thompson, Autophagy: Basic principles and relevance to disease. Annu. Rev. Pathol. 3 427-455 (2008). doi: 10.1146/annurev.pathmechdis. 2.010506.091842; pmid: 18039129

25. V. M. Hubbard et al., Macroautophagy regulates energy metabolism during effector T cell activation. J. Immunol. 185, 7349-7357 (2010). doi: 10.4049/jimmunol. 1000576; pmid: 21059894

26. H. H. Pua, I. Dzhagalov, M. Chuck, N. Mizushima, Y. W. He, A critical role for the autophagy gene Atg5 in T cell survival and proliferation. J. Exp. Med. 204, 25-31 (2007). doi: 10.1084/jem.20061303; pmid: 17190837

27. C. H. Chang et al., Posttranscriptional control of T cell effector function by aerobic glycolysis. Cell 153, 1239-1251 (2013). doi: 10.1016/j.cell.2013.05.016; pmid: 23746840

28. G. Chaudhri, I. A. Clark, N. H. Hunt, W. B. Cowden, R. Ceredig, Effect of antioxidants on primary alloantigeninduced T cell activation and proliferation. J. Immunol. 137, 2646-2652 (1986).pmid: 2944959

29. S. Devadas, L. Zaritskaya, S. G. Rhee, L. Oberley, M. S. Williams, Discrete generation of superoxide and hydrogen peroxide by $T$ cell receptor stimulation: Selective regulation of mitogen-activated protein kinase activation and fas ligand expression. J. Exp. Med. 195, 59-70 (2002). doi: 10.1084/jem.20010659; pmid: 11781366

30. S. H. Jackson, S. Devadas, J. Kwon, L. A. Pinto, M. S. Williams, T cells express a phagocyte-type NADPH oxidase that is activated after $\mathrm{T}$ cell receptor stimulation. Nat. Immunol. 5, 818-827 (2004). doi: 10.1038/ ni1096; pmid: 15258578

31. L. A. Sena et al., Mitochondria are required for antigen-specific $T$ cell activation through reactive oxygen species signaling. Immunity 38, 225-236 (2013). doi: 10.1016/j.immuni.2012.10.020; pmid: 23415911

32. S. Krauss, M. D. Brand, F. Buttgereit, Signaling takes a breath-New quantitative perspectives on bioenergetics and signal transduction. Immunity 15, 497-502 (2001). doi: 10.1016/S1074-7613(01)00205-9; pmid: 11672532

33. R. G. Jones et al., The proapoptotic factors Bax and Bak regulate $\mathrm{T}$ Cell proliferation through control of endoplasmic reticulum $\mathrm{Ca}(2+)$ homeostasis. Immunity 27, 268-280 (2007). doi: 10.1016/j.immuni.2007. 05.023; pmid: 17692540

34. R. D. Michalek et al., Cutting edge: Distinct glycolytic and lipid oxidative metabolic programs are essential for effector and regulatory CD4+ T cell subsets. J. Immunol. 186, 3299-3303 (2011). doi: 10.4049/jimmunol. 1003613; pmid: 21317389

35. L. Z. Shi et al., HIF1 $\alpha$-dependent glycolytic pathway orchestrates a metabolic checkpoint for the differentiation of $\mathrm{T}_{\mathrm{H}} 17$ and $\mathrm{T}_{\text {reg }}$ cells. J. Exp. Med. 208, 1367-1376 (2011). doi: 10.1084/jem.20110278 pmid: 21708926

36. E. V. Dang et al., Control of $\mathrm{T}(\mathrm{H}) 17 / \mathrm{T}(\mathrm{reg})$ balance by hypoxia-inducible factor 1 . Cell 146, 772-784 (2011). doi: 10.1016/j.cell.2011.07.033; pmid: 21871655

37. C. Wu et al., Induction of pathogenic $T_{H} 17$ cells by inducible salt-sensing kinase SGK1. Nature 496, 513-517 (2013). doi: 10.1038/nature11984; pmid: 23467085

38. M. Kleinewietfeld et al., Sodium chloride drives autoimmune disease by the induction of pathogenic $T_{H} 17$ cells. Nature 496, 518-522 (2013). doi: 10.1038/ nature11868; pmid: 23467095

39. P. M. Smith et al., The microbial metabolites, short-chain fatty acids, regulate colonic $T_{\text {reg }}$ cell homeostasis. Science 341, 569-573 (2013); 10.1126/science.1241165. doi: 10.1126/science.1241165; pmid: 23828891

40. P. S. Ward, C. B. Thompson, Metabolic reprogramming: A cancer hallmark even Warburg did not anticipate. Cancer Cell 21, 297-308 (2012). doi: 10.1016/ j.ccr.2012.02.014; pmid: 22439925
41. C. A. Doughty et al., Antigen receptor-mediated changes in glucose metabolism in B lymphocytes: Role of phosphatidylinositol 3-kinase signaling in the glycolytic control of growth. Blood 107, 4458-4465 (2006). doi: 10.1182/blood-2005-12-4788; pmid: 16449529

42. C. M. Krawczyk et al., Toll-like receptor-induced changes in glycolytic metabolism regulate dendritic cell activation. Blood 115, 4742-4749 (2010). doi: 10.1182/ blood-2009-10-249540; pmid: 20351312

43. A. Haschemi et al., The sedoheptulose kinase CARKL directs macrophage polarization through control of glucose metabolism. Cell Metab. 15, 813-826 (2012). doi: 10.1016/j.cmet.2012.04.023; pmid: 22682222

44. R. J. DeBerardinis, N. Sayed, D. Ditsworth, C. B. Thompson, Brick by brick: Metabolism and tumor cell growth. Curr. Opin. Genet. Dev. 18, 54-61 (2008). doi: 10.1016/j.gde.2008.02.003; pmid: 18387799

45. K. E. Wellen, C. B. Thompson, A two-way street: Reciprocal regulation of metabolism and signalling. Nat. Rev. Mol. Cell Biol. 13, 270-276 (2012). pmid: 22395772

46. R. J. DeBerardinis, J. J. Lum, G. Hatzivassiliou, C. B. Thompson, The biology of cancer: Metabolic reprogramming fuels cell growth and proliferation. Cell Metab. 7, 11-20 (2008). doi: 10.1016/j.cmet.2007. 10.002; pmid: 18177721

47. G. Hatzivassiliou et al., ATP citrate lyase inhibition can suppress tumor cell growth. Cancer Cell 8, 311-321 (2005). doi: 10.1016/j.ccr.2005.09.008; pmid: 16226706

48. Y. Kidani et al., Sterol regulatory element-binding proteins are essential for the metabolic programming of effector T cells and adaptive immunity. Nat. Immunol. 14, 489-499 (2013). doi: 10.1038/ni.2570 pmid: 23563690

49. Q. Wang et al., Acetylation of metabolic enzymes coordinates carbon source utilization and metabolic flux. Science 327, 1004-1007 (2010). doi: 10.1126/ science.1179687; pmid: 20167787

50. S. Zhao et al., Regulation of cellular metabolism by protein lysine acetylation. Science 327, 1000-1004 (2010). doi: 10.1126/science.1179689; pmid: 20167786

51. K. E. Wellen et al., ATP-citrate lyase links cellular metabolism to histone acetylation. Science 324 1076-1080 (2009). doi: 10.1126/science.1164097; pmid: 19461003

52. M. Griffiths, D. Keast, The effect of glutamine on murine splenic leukocyte responses to $T$ and $B$ cell mitogens. Immunol. Cell Biol. 68, 405-408 (1990). doi: 10.1038/ icb.1990.54; pmid: 2097296

53. A. Le et al., Glucose-independent glutamine metabolism via TCA cycling for proliferation and survival in B cells. Cell Metab. 15, 110-121 (2012). doi: 10.1016/ j.cmet.2011.12.009; pmid: 22225880

54. S. Mazurek, Pyruvate kinase type M2: A key regulator of the metabolic budget system in tumor cells. Int. J. Biochem. Cell Biol. 43, 969-980 (2011). doi: 10.1016/ j.biocel.2010.02.005; pmid: 20156581

55. S. Marjanovic, I. Eriksson, B. D. Nelson, Expression of a new set of glycolytic isozymes in activated human peripheral lymphocytes. Biochim. Biophys. Acta 1087, 1-6 (1990). doi: 10.1016/0167-4781(90)90113-G; pmid: 2169315

56. D. Anastasiou et al., Pyruvate kinase M2 activators promote tetramer formation and suppress tumorigenesis. Nat. Chem. Biol. 8, 839-847 (2012). doi: 10.1038/ nchembio.1060; pmid: 22922757

57. H. R. Christofk et al., The M2 splice isoform of pyruvate kinase is important for cancer metabolism and tumour growth. Nature 452, 230-233 (2008) doi: 10.1038/nature06734; pmid: 18337823

58. H. R. Christofk, M. G. Vander Heiden, N. Wu, J. M. Asara, L. C. Cantley, Pyruvate kinase M2 is a phosphotyrosinebinding protein. Nature 452, 181-186 (2008). doi: 10.1038/nature06667; pmid: 18337815

59. T. Hitosugi et al., Tyrosine phosphorylation inhibits PKM2 to promote the Warburg effect and tumor growth. Sci. Signal. 2, ra73 (2009). doi: 10.1126/scisignal.2000431; pmid: 19920251

60. C. Kung et al., Small molecule activation of PKM2 in cancer cells induces serine auxotrophy. Chem. Biol. 19
1187-1198 (2012). doi: 10.1016/j.chembiol.2012.07.021 pmid: 22999886

61. W. Luo et al., Pyruvate kinase M2 is a PHD3-stimulated coactivator for hypoxia-inducible factor 1 . Cell 145 732-744 (2011). doi: 10.1016/j.cell.2011.03.054; pmid: 21620138

62. W. Yang et al., PKM2 phosphorylates histone $\mathrm{H} 3$ and promotes gene transcription and tumorigenesis. Cell 150, 685-696 (2012). doi: 10.1016/j.cell.2012.07.018 pmid: 22901803

63. X. Gao, H. Wang, ]. ]. Yang, X. Liu, Z. R. Liu, Pyruvate kinase $M 2$ regulates gene transcription by acting as a protein kinase. Mol. Cell 45, 598-609 (2012). doi: 10.1016/j.molcel.2012.01.001; pmid: 22306293

64. B. Chaneton et al., Serine is a natural ligand and allosteric activator of pyruvate kinase M2. Nature 491 458-462 (2012). doi: 10.1038/nature11540; pmid: 23064226

65. J. W. Locasale et al., Phosphoglycerate dehydrogenase diverts glycolytic flux and contributes to oncogenesis. Nat. Genet. 43, 869-874 (2011). doi: 10.1038/ng.890 pmid: 21804546

66. R. Possemato et al., Functional genomics reveal that the serine synthesis pathway is essential in breast cancer. Nature 476, 346-350 (2011). doi: 10.1038/ nature10350; pmid: 21760589

67. 0. D. Maddocks et al., Serine starvation induces stress and p53-dependent metabolic remodelling in cance cells. Nature 493, 542-546 (2013). doi: 10.1038/ nature11743; pmid: 23242140

68. L. M. Nilsson et al., Mouse genetics suggests cell-context dependency for Myc-regulated metabolic enzymes during tumorigenesis. PLoS Genet. 8, e1002573 (2012). doi: 10.1371/journal.pgen.1002573; pmid: 22438825

69. R. J. DeBerardinis et al., Beyond aerobic glycolysis: Transformed cells can engage in glutamine metabolism that exceeds the requirement for protein and nucleotide synthesis. Proc. Natl. Acad. Sci. U.S.A. 104 19345-19350 (2007). doi: 10.1073/pnas.0709747104 pmid: 18032601

70. A. R. Mullen et al., Reductive carboxylation supports growth in tumour cells with defective mitochondria. Nature 481, 385-388 (2012). pmid: 22101431

71. C. M. Metallo et al., Reductive glutamine metabolism by IDH1 mediates lipogenesis under hypoxia. Nature 481, 380-384 (2012). pmid: 22101433

72. D. R. Wise et al., Hypoxia promotes isocitrate dehydrogenase-dependent carboxylation of $\alpha$-ketoglutarate to citrate to support cell growth and viability. Proc. Natl. Acad. Sci. U.S.A. 108 19611-19616 (2011). doi: 10.1073/pnas.1117773108 pmid: 22106302

73. E. N. McNamee, D. Korns Johnson, D. Homann, E. T. Clambey, Hypoxia and hypoxia-inducible factors as regulators of $\mathrm{T}$ cell development, differentiation, and function. Immunol. Res. 55, 58-70 (2013). doi: 10.1007/s12026-012-8349-8; pmid: 22961658

74. H. K. Eltzschig, P. Carmeliet, Hypoxia and inflammation. N. Engl. J. Med. 364, 656-665 (2011). doi: 10.1056/ NEJMra0910283; pmid: 21323543

75. H. Nakamura et al., TCR engagement increases hypoxia-inducible factor-1 alpha protein synthesis via rapamycin-sensitive pathway under hypoxic conditions in human peripheral T cells. J. Immunol. 174, 7592-7599 (2005). pmid: 15944259

76. E. T. Clambey et al., Hypoxia-inducible factor-1 alphadependent induction of FoxP3 drives regulatory T-cell abundance and function during inflammatory hypoxia of the mucosa. Proc. Natl. Acad. Sci. U.S.A. 109, E2784-E2793 (2012). doi: 10.1073/pnas.1202366109; pmid: 22988108

77. D. W. Parsons et al., An integrated genomic analysis of human glioblastoma multiforme. Science 321, 1807-1812 (2008); 10.1126/science.1164382. doi: 10.1126/science.1164382; pmid: 18772396

78. L. Dang et al., Cancer-associated IDH1 mutations produce 2-hydroxyglutarate. Nature 462, 739-744 (2009). doi: 10.1038/nature08617; pmid: 19935646

79. M. E. Figueroa et al., Leukemic IDH1 and IDH2 mutations result in a hypermethylation phenotype, disrupt TET2 
function, and impair hematopoietic differentiation. Cancer Cell 18, 553-567 (2010). doi: 10.1016/ j.ccr.2010.11.015; pmid: 21130701

80. C. Lu et al., IDH mutation impairs histone demethylation and results in a block to cell differentiation. Nature 483 474-478 (2012). doi: 10.1038/nature10860 pmid: 22343901

81. W. Xu et al., Oncometabolite 2-hydroxyglutarate is a competitive inhibitor of $\alpha$-ketoglutarate-dependent dioxygenases. Cancer Cell 19, 17-30 (2011). doi: 10.1016/j.ccr.2010.12.014; pmid: 21251613

82. S. Turcan et al., IDH1 mutation is sufficient to establish the glioma hypermethylator phenotype. Nature 483, 479-483 (2012). doi: 10.1038/nature10866; pmid: 22343889

83. D. G. Hardie, F. A. Ross, S. A. Hawley, AMPK: A nutrient and energy sensor that maintains energy homeostasis Nat. Rev. Mol. Cell Biol. 13, 251-262 (2012) doi: 10.1038/nrm3311; pmid: 22436748

84. B. Xiao et al., Structure of mammalian AMPK and its regulation by ADP. Nature 472, 230-233 (2011). doi: 10.1038/nature09932; pmid: 21399626

85. ]. S. Oakhill et al., AMPK is a direct adenylate charge-regulated protein kinase. Science 332, 1433-1435 (2011). doi: 10.1126/science.1200094; pmid: 21680840

86. F. Buttgereit, M. D. Brand, A hierarchy of ATP-consuming processes in mammalian cells. Biochem. J. 312, 163-167 (1995). pmid: 7492307

87. P. Tamás et al., Regulation of the energy sensor AMP-activated protein kinase by antigen receptor and Ca2+ in T lymphocytes. J. Exp. Med. 203, 1665-1670 (2006). doi: 10.1084/jem.20052469; pmid: 16818670

88. N. J. Maclver et al., The liver kinase B1 is a central regulator of T cell development, activation, and metabolism. J. Immunol. 187, 4187-4198 (2011). doi: 10.4049/jimmunol.1100367; pmid: 21930968

89. B. Faubert et al., AMPK is a negative regulator of the Warburg effect and suppresses tumor growth in vivo. Cell Metab. 17, 113-124 (2013). doi: 10.1016/ j.cmet.2012.12.001; pmid: 23274086

90. P. Tamás et al., LKB1 is essential for the proliferation of T-cell progenitors and mature peripheral T cells. Eur. J. Immunol. 40, 242-253 (2010). doi: 10.1002/ eji.200939677; pmid: 19830737

91. R. G. Jones et al., AMP-activated protein kinase induces p53-dependent metabolic checkpoint. Mol. Cell 18, 283-293 (2005). doi: 10.1016/j.molcel.2005.03.027; pmid: 15866171

92. ]. Rolf et al., AMPK $\alpha 1$ : A glucose sensor that controls CD8 T-cell memory. Eur. J. Immunol. 43, 889-896 (2013). doi: 10.1002/eji.201243008; pmid: 23310952

93. N. L. Alderson et al., S-(2-Succinyl)cysteine: A novel chemical modification of tissue proteins by a Krebs cycle intermediate. Arch. Biochem. Biophys. 450, 1-8 (2006). doi: 10.1016/j.abb.2006.03.005; pmid: 16624247

94. C. Bardella et al., Aberrant succination of proteins in fumarate hydratase-deficient mice and HLRCC patients is a robust biomarker of mutation status. J. Pathol. 225 4-11 (2011). doi: 10.1002/path.2932; pmid: 21630274

95. J. Adam et al., Renal cyst formation in Fh1-deficient mice is independent of the Hif/Phd pathway: Roles for fumarate in KEAP1 succination and Nrf2 signaling.
Cancer Cell 20, 524-537 (2011). doi: 10.1016/ j.ccr.2011.09.006; pmid: 22014577

96. W. G. Kaelin Jr., Cancer and altered metabolism: Potential importance of hypoxia-inducible factor and 2-oxoglutarate-dependent dioxygenases. Cold Spring Harb. Symp. Quant. Biol. 76, 335-345 (2011). doi: 10.1101/sqb.2011.76.010975; pmid: 22089927

97. G. L. Semenza, Hypoxia-inducible factors in physiology and medicine. Cell 148, 399-408 (2012). doi: 10.1016/ j.cell.2012.01.021; pmid: 22304911

98. ]. A. Losman, W. G. Kaelin Jr., What a difference a hydroxyl makes: Mutant IDH, (R)-2-hydroxyglutarate, and cancer. Genes Dev. 27, 836-852 (2013). doi: 10.1101/ gad.217406.113; pmid: 23630074

99. P. S. Ward et al., The common feature of leukemiaassociated IDH1 and IDH2 mutations is a neomorphic enzyme activity converting alpha-ketoglutarate to 2-hydroxyglutarate. Cancer Cell 17, 225-234 (2010). doi: 10.1016/j.ccr.2010.01.020; pmid: 20171147

100. ]. S. Isaacs et al., HIF overexpression correlates with biallelic loss of fumarate hydratase in renal cancer: Novel role of fumarate in regulation of HIF stability. Cancer Cell 8, 143-153 (2005). doi: 10.1016/j.ccr.2005.06.017; pmid: 16098467

101. M. A. Selak et al., Succinate links TCA cycle dysfunction to oncogenesis by inhibiting HIF-alpha prolyl hydroxylase. Cancer Cell 7, 77-85 (2005). doi: 10.1016/ j.ccr.2004.11.022; pmid: 15652751

102. G. M. Tannahill et al., Succinate is an inflammatory signal that induces IL-1 $\beta$ through HIF-1 $\alpha$. Nature 496, 238-242 (2013). doi: 10.1038/nature11986; pmid: 23535595

103. Y. Kanno, G. Vahedi, K. Hirahara, K. Singleton, ]. ]. O'Shea, Transcriptional and epigenetic control of T helper cell specification: Molecular mechanisms underlying commitment and plasticity. Annu. Rev. Immunol. 30, 707-731 (2012). doi: 10.1146/annurevimmunol-020711-075058; pmid: 22224760

104. M. W. Hentze, T. Preiss, The REM phase of gene regulation. Trends Biochem. Sci. 35, 423-426 (2010). doi: 10.1016/j.tibs.2010.05.009; pmid: 20554447

105. A. Castello et al., Insights into RNA biology from an atlas of mammalian mRNA-binding proteins. Cell 149, 1393-1406 (2012). doi: 10.1016/j.cell.2012.04.031; pmid: 22658674

106. J. Cieśla, Metabolic enzymes that bind RNA: Yet another level of cellular regulatory network? Acta Biochim. Pol. 53, 11-32 (2006).pmid: 16410835

107. M. W. Hentze, P. Argos, Homology between IRE-BP, a regulatory RNA-binding protein, aconitase, and isopropylmalate isomerase. Nucleic Acids Res. 19, 1739-1740 (1991). doi: 10.1093/nar/19.8.1739; pmid: 1903202

108. T. A. Rouault, C. D. Stout, S. Kaptain, ]. B. Harford, R. D. Klausner, Structural relationship between an ironregulated RNA-binding protein (IRE-BP) and aconitase: Functional implications. Cell 64, 881-883 (1991) doi: 10.1016/0092-8674(91)90312-M; pmid: 2001588

109. M. W. Hentze, M. U. Muckenthaler, B. Galy, C. Camaschella, Two to tango: Regulation of Mammalian iron metabolism. Cell 142, 24-38 (2010). doi: 10.1016/ j.cell.2010.06.028; pmid: 20603012

110. A. Constable, S. Quick, N. K. Gray, M. W. Hentze, Modulation of the RNA-binding activity of a regulatory protein by iron in vitro: Switching between enzymatic and genetic function? Proc. Natl. Acad. Sci. U.S.A. 89, 4554-4558 (1992). doi: 10.1073/pnas.89.10.4554; pmid: 1584791

111. E. Nagy, W. F. C. Rigby, Glyceraldehyde-3-phosphate dehydrogenase selectively binds AU-rich RNA in the NAD (+)-binding region (Rossmann fold). J. Biol. Chem. 270, 2755-2763 (1995). doi: 10.1074/jbc.270.6.2755 pmid: 7531693

112. E. Nagy et al., Identification of the NAD(+)-binding fold of glyceraldehyde-3-phosphate dehydrogenase as a nove RNA-binding domain. Biochem. Biophys. Res. Commun. 275, 253-260 (2000). doi: 10.1006/bbrc.2000.3246; pmid: 10964654

113. A. Colell et al., GAPDH and autophagy preserve survival after apoptotic cytochrome c release in the absence of caspase activation. Cell 129, 983-997 (2007). doi: 10.1016/j.cell.2007.03.045; pmid: 17540177

114. C. Tristan, N. Shahani, T. W. Sedlak, A. Sawa, The diverse functions of GAPDH: Views from different subcellular compartments. Cell. Signal. 23, 317-323 (2011). doi: 10.1016/j.cellsig.2010.08.003; pmid: 20727968

115. R. J. DeBerardinis, C. B. Thompson, Cellular metabolism and disease: What do metabolic outliers teach us? Cell 148, 1132-1144 (2012). doi: 10.1016/ j.cell.2012.02.032; pmid: 22424225

116. Y. P. Hung, J. G. Albeck, M. Tantama, G. Yellen, Imaging cytosolic NADH-NAD(+) redox state with a genetically encoded fluorescent biosensor. Cell Metab. 14, 545-554 (2011). doi: 10.1016/j.cmet.2011.08.012; pmid: 21982714

117. P. Tsou, B. Zheng, C. H. Hsu, A. T. Sasaki, L. C. Cantley, A fluorescent reporter of AMPK activity and cellular energy stress. Cell Metab. 13, 476-486 (2011). doi: 10.1016/ j.cmet.2011.03.006; pmid: 21459332

118. I. Marin-Valencia et al., Analysis of tumor metabolism reveals mitochondrial glucose oxidation in genetically diverse human glioblastomas in the mouse brain in vivo. Cell Metab. 15, 827-837 (2012). doi: 10.1016/ j.cmet.2012.05.001; pmid: 22682223

119. I. Marin-Valencia et al., Glucose metabolism via the pentose phosphate pathway, glycolysis and Krebs cycle in an orthotopic mouse model of human brain tumors. NMR Biomed. 25, 1177-1186 (2012). doi: 10.1002 nbm.2787; pmid: 22383401

120. A. L. Mellor, D. H. Munn, IDO expression by dendritic cells: Tolerance and tryptophan catabolism. Nat. Rev. Immunol. 4, 762-774 (2004). doi: 10.1038/nri1457; pmid: 15459668

121. K. Fischer et al., Inhibitory effect of tumor cell-derived lactic acid on human T cells. Blood 109, 3812-3819 (2007) doi: 10.1182/blood-2006-07-035972; pmid: 17255361

Acknowledgments: We thank ]. Blagih, E. Clambey, M. Hentze, C. Krawczyk, M. Vander Heiden, and members of the Jones and Pearce laboratories for insight and comments on this manuscript and L. Donnelly and M. Maslowska for administrative help. This work was supported by grants to R.G.J. from the Canadian Institute for Health Research (MOP-93799 and a New Investigator Career Award) and the Arthritis Society of Canada and by grants to E.L.P. from National Institute of Allergy and Infectious Diseases (Al091965) and National Cancer Institute (CA158823).

10.1126/science. 1242454 


\section{Science}

\section{Fueling Immunity: Insights into Metabolism and Lymphocyte Function}

Erika L. Pearce, Maya C. Poffenberger, Chih-Hao Chang and Russell G. Jones

Science 342 (6155), 1242454.

DOI: $10.1126 /$ science.1242454

\section{Lymphocyte Metabolism}

Lymphocytes are highly dynamic cells, undergoing extensive proliferation upon infection and then reducing in number upon pathogen clearance. Lymphocytes also circulate through many different tissue environments that vary in their nutrient and oxygen availability. Recent studies have revealed changes in metabolic programming that facilitate this dynamic behavior. How these changes occur and the specific effects that they have on lymphocyte function and on the ultimate outcome of an infection are not well understood. Pearce et al. (1242454) review recent progress in this area, suggest how parallels might be found in studying the metabolic changes seen in tumor cells, and propose challenges for the future.

ARTICLE TOOLS

http://science.sciencemag.org/content/342/6155/1242454

RELATED

CONTENT

REFERENCES

PERMISSIONS http://stke.sciencemag.org/content/sigtrans/8/370/re4.full http://stke.sciencemag.org/content/sigtrans/8/396/ra97.full http://stke.sciencemag.org/content/sigtrans/8/396/pc25.full

This article cites 121 articles, 35 of which you can access for free http://science.sciencemag.org/content/342/6155/1242454\#BIBL

http://www.sciencemag.org/help/reprints-and-permissions

Use of this article is subject to the Terms of Service

Science (print ISSN 0036-8075; online ISSN 1095-9203) is published by the American Association for the Advancement of Science, 1200 New York Avenue NW, Washington, DC 20005. The title Science is a registered trademark of AAAS.

Copyright $@ 2013$, American Association for the Advancement of Science 